\title{
Generalized Path-Permutation Codes for Reliable End-to-End Networking of Opportunistic Links
}

\author{
I-Wei Lai, Member, IEEE, Jhih-Wei Shih, Student Member, IEEE, \\ Kwang-Cheng Chen, Fellow, IEEE, and Ezio Biglieri, Life Fellow, IEEE
}

\begin{abstract}
Opportunistic links are emerging as a novel technology for state-of-the-art wireless networks, such as cognitive radio networks, energy-harvesting networks, and low-latency vehicular networks. To increase the reliability of these networks, path-permutation codes (PPCs) were advocated in [1]. These codes are based on a virtual multiple-input multipleoutput (MIMO) model on the session/network layer, and have been tailored for increased reliability of opportunistic links. By exploiting multipath-multihop routes, PPCs access one relay path at a time while repeatedly transmitting the same QAM symbol. In this paper, we introduce a generalized PPC scheme which simultaneously accesses multiple relay paths and comprises multiple QAM symbols in the packet. Phase rotations are also introduced in the PPC codewords for additional performance improvement. Numerical simulation results validate our analyses and demonstrate the superior performance of this novel coding scheme, i.e., better error rate performance, higher throughput, and stronger robustness to transmission outages.
\end{abstract}

Index Terms-Multiple-input multiple-output (MIMO), virtual MIMO, low-latency vehicular network, cognitive radio network, energy-harvesting network, proactive open-loop communication, ultra-low latency, erasure channels, path-permutation codes.

\section{INTRODUCTION}

Stringent performance requirements of modern wireless networks may force dramatic changes in design criteria. For example, the safety needs of vehicular networks [2], [3] may call for a reduction of latency from hundreds of msec to about 1 msec: as a consequence, traditional closed-loop physicallayer communication involving power control, channel estimation, positive/negative acknowledgment of a received/missing packet, etc. [4]-[6] may not be adequate to guarantee that ultra-low latency is achieved.

A natural solution to the problems connected with latency reduction is grounded on open-loop (or proactive) communication: this speeds up data transfer to upper protocol layers by forsaking end-to-end feedback and retransmission in physical layer [7]. Other techniques, in addition to open-loop transmission, have also been recently advocated [3], [8], [9]. Among these, virtual cells allow the mobile user (autonomous vehicle)

The work of I-Wei Lai was supported by Ministry of Science and Technology, Taiwan, under Grant MOST 108-2221-E-003-002-MY3. K.-C. Chen's research is supported in part by the Cyber Florida. The work of Ezio Biglieri was supported by the H2020 Framework Programme/ERC grant 694974. I-Wei Lai and Jhih-Wei Shih are with the Department of Electrical Engineering, National Taiwan Normal University, Taipei, Taiwan; KwangCheng Chen is with the Department of Electrical Engineering, University of South Florida, Tampa, FL 33620 USA; E. Biglieri is with Universitat Pompeu Fabra, Barcelona, Spain. E-mail: iweilai@ntnu.edu.tw, kwangcheng@usf.edu, e.biglieri@ieee.org to modify proactively the topology of a network through proper selection of the access points in its close proximity. This procedure would avoid the latency increase intrinsic in the handover mechanism. Such proactive communication greatly improves the latency in heterogeneous cloud radio access network (H-CRAN) [10]. Specifically, by proactively associating up to three access points on average, the latency can be reduced from more than 1 second to the level of milliseconds. However, since the network infrastructure must be made privy to the association of a virtual cell to the mobile user, which may be a problem especially under the high mobility scenario [9], anticipatory mobility management has been advocated. This exploits current location and association information to predict the access points that will be associated with the mobile user in the near future [11].

These techniques, which can be categorized under the rubric of opportunistic links, are vulnerable to transmission outages: in fact, deep fading and interference affect open-loop communications because of the absence of control and feedback mechanisms. Moreover, since the same radio resource unit may be accessed by mobile users in different virtual cells, multiple access in the air may lose orthogonality, and hence causes collisions. In addition, when anticipatory mobility management fails to predict the correct positions of mobile users, downlink data are directed to the wrong access points, which causes packet loss and collisions.

In the following, we describe a novel design procedure for low-latency networks based on proactive communication. Our solution achieves reliable end-to-end networking of opportunistic links using the concept of virtual multiple-input multiple-output (MIMO) on the network/session layer, as described in [12]. Here, the source node transmits data through a multihop-multipath route [13], as commonly used for example in vehicular networks. The mobile user transmits uplink signals to the network infrastructure via multiple access points, with multiple relay paths interpreted as virtual transmit antennas in a virtual MIMO system. By doing so, the data can be encoded along path and time coordinates using path-permutation codes (PPC) [1].

These codes, as designed for a virtual MIMO framework, access one relay path at a time using a suitable permutation pattern [1]. Fig. 1(a) illustrates a simple design of the original version of PPC using two relay paths and two time instants. Here, the source node transmits one bit by choosing one out of two permutation vectors, which in this example are 


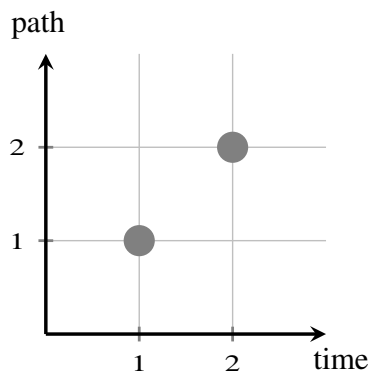

(a) Original version

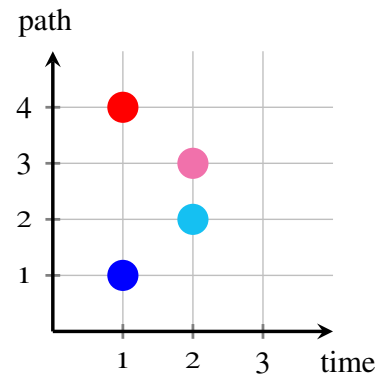

(b) Generalized version
Fig. 1. Time-path plane representation of the transmission using PPC.

$[1,2]^{\top}$ and $[2,1]^{\top}$, where $(\cdot)^{\top}$ denotes transpose. To transmit bit ' 0 ', permutation $[1,2]^{\top}$ is selected, which means that a QAM symbol is transmitted at time 1 on path 1 , and at time 2 on path 2 . Vice versa, to transmit bit ' 1 ' a QAM symbol is transmitted at time 1 on path 2 , and at time 2 on path 1 , as described by permutation $[2,1]^{\top}$. In general, a PPC scheme is identified by a set of permutation vectors and a single QAM symbol, which is detected at the destination node (privy to the encoding scheme) for data recovery. PPC exploits the permutation to convey additional information bits and to allow the signal hops among paths to mitigate the performance degradation caused by the path/time unavailability. Recently, the term index modulation [14] was used to describe techniques using the indices of the communication entities to transmit data: for example, spatial modulation of the physical-layer MIMO system [15]. PPC can be regarded as a novel implementation of the concept of index modulation, and the utilization of permutations may improve the performance of other index modulation techniques. For example, in [16] spatial permutation modulation is investigated by integrating permutations and spatial modulation.

In our work, we describe a two-way extension of PPC. First, PPC is generalized so as to allow the source node to access simultaneously $K$ relay paths, as illustrated in a simple example by Fig. 1(b) with $K=2$. Second, multiple $Q A M$ symbols are allowed to be transmitted in the 2-dimensional time-path plane. Further, suitable phase rotations of QAM symbols improve both transmit diversity and robustness to erasures. This generalization is conceptually simple and yields an effective way of realizing reliable end-to-end low-latency networking with proactive open-loop communication. Yet, its design leaves some challenges to overcome. For example, if more paths are accessed using different QAM symbols, the throughput is increased, but the reliability may be reduced due to lower diversity and added interference. The design space of such generalized PPC increases greatly, resulting into a complex interplay of transmission parameters. To resolve these issues, we conduct extensive theoretical analyses involving error rate, diversity, and error floor. These analyses enable fundamental understanding of generalized PPC, allow fast and systematic design space exploration, and disclose suitable design guidelines. For example, QAM diversity depends on the repetition along the time coordinate, and thus for lower erasure probability various QAM symbols should be transmitted at various relay paths to enhance throughput, while for lower error rate lower-order modulation alphabets should be adopted. Also, when transmission outages occur frequently (high erasure probability), repetition of QAM symbols over various relay paths lowers the error floor. The improvements obtained by introducing phase rotations are illustrated using a combination of theoretical analysis and numerical experiments. Finally, since the number of usable permutation vectors increases with the increase of the number of accessed paths, we formulate the selection of PPC permutation vectors as a hierarchical optimization, and propose a two-phase greedy algorithm to design this permutation set. In summary, the major contributions of this work are enumerated below:

(1) A generalization of PPC allowing simultaneous access of multiple relay paths and transmission of multiple QAM symbols.

(2) Introduction of phase rotations of the transmitted QAM symbols to lower the error rate.

(3) Illustration of the improvement of error rate, throughput, and robustness to random outages (lower error floor) allowed by generalized PPC, which thus enable reliable end-to-end networking of opportunistic links.

(4) Systematic design space exploration and design guidelines of generalized PPC.

This paper is organized as follows. Section II introduces the scenario of multipath multihop end-to-end networking, the fading erasure channel model for opportunistic links, the formulation of the virtual MIMO framework, and the conventional PPC. Section III elaborates on the generalizations of end-to-end PPC networking and on the algorithm for permutation-set optimization. Comprehensive theoretical analyses are conducted in Section IV. The rotation technique and the associated performance analysis are addressed in Section V. Section VI provides numerical results validating our analyses, and demonstrating the performance of generalized end-to-end PPC networking and of the rotation technique in a general multihop-multipath networking of opportunistic links, with the applications like low-latency vehicular networks and cognitive radio networks. Finally, conclusions are drawn and future work is suggested in Section VII.

\section{End-to-End Virtual MiMO Networking in Fading Erasure Channel}

Throughout this paper we define a link as the connection between two nodes, and a path as the end-to-end connection between source and destination nodes. For simplicity's sake, we assume that all paths comprise the same number $L$ of low-latency opportunistic links [17]. The topology of this network [1], [18]-[21] is illustrated by the directed-graph model depicted in Fig. 2.

The routing algorithms like ad hoc on-demand multipath distance vector (AOMDV) can be used to establish the multichip-multipath route where the availabilities of links in different paths are independent. This way, the $R$ link disjoint paths are allowed to be simultaneously accessed [18], [19], [22]. The source node transmits coded packets to the destination node through multiple relay paths, where each relay node uses an amplify-and-forward protocol. During transmission, inter-path interference occurs at relay nodes, but 


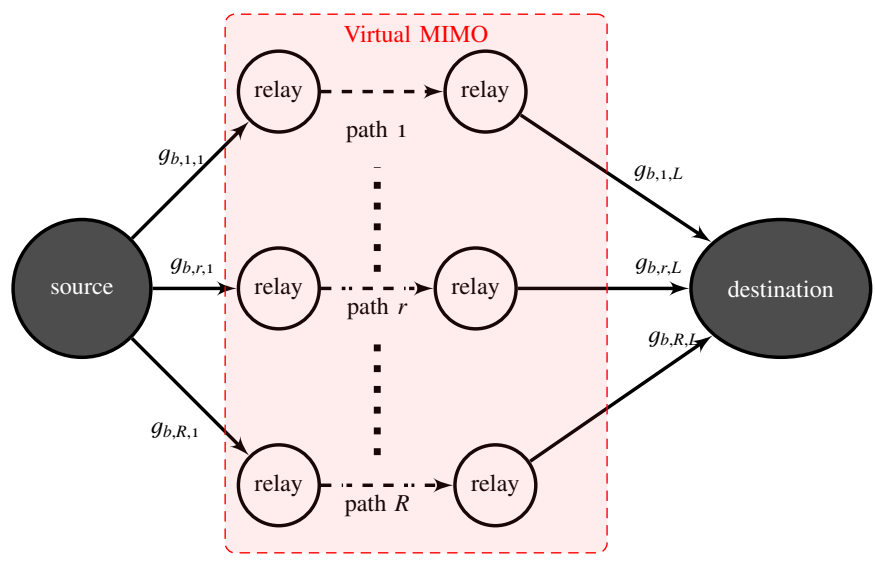

Fig. 2. The directed-graph model of the disjoint multipath relay networks. The nodes ' $S$ ', 'R', and ' $D$ ' represent source, relay, and destination node, respectively. In vehicular networks, relay nodes model access points.

can be generally mitigated, if not avoided, by using directional antennas or selection mechanisms [23]-[25]. Within scenarios like low-latency vehicular networks, where collisions are inevitable since vehicles simultaneously access the same radio source of the same access point, this severe interference may result in transmission outages. These collisions can be studied using erasure channel models, and mitigated using virtual MIMO coding techniques on the network/session layer [12]. Specifically, a fading erasure channel model tailored to opportunistic links is used. Opportunistic links in different network applications can be characterized by various erasure statistics. For example, in energy-harvesting network, when running out of harvested energy, we have consecutive erasures whose statistics depend on the statistics of the harvested energy [21].

\section{A. Erasure Model For Opportunistic Links}

During end-to-end networking, the destination may fail to receive the data due to a transmission outage caused by the unreliable opportunistic links. These outages, being erasures, can be modeled by Bernoulli-distributed random variables [12], [20], [26]. In particular, we define the erasure matrix $\mathbf{V} \in\{0,1\}^{M \times R}$, where $M$ is the number of discrete time instants used to transmit a coded packet. The $(m, r)$ th entry $v_{m, r}$ is a Bernoulli random variable [27] that takes value ' 0 ' when an erasure occurs at the $r$ th path and time $m$, and otherwise value ' 1 ' indicating path availability. We assume that the entries of $\mathbf{V}$ are statistically independent.

The opportunistic links can be described by the erasure matrix whose statistics depend on the outage sources. For example, in low-latency vehicular networks with proactive open-loop communication, since the vehicles proactively and autonomously associate the access points nearby, different users may concurrently access the same radio resource. If the mobile users are capable of spectrum sensing, as with cognitive radios, the packet is buffered until the spectrum is available or the accumulated latency exceeds a maximum value. If the latter event takes place, the packet is discarded and an erasure occurs. The probability of such packet expiration is characterized by first defining the number of sensing attempts $W$, i.e., the ratio of packet lifetime to the sensing interval. This indicates that each discrete time instant $m$ includes $W$ sensing intervals. These are used for the packet to be successively propagated through the $L$ opportunistic links in a path. This process is equivalent to a Bernoulli trial, where the end-to-end delay (normalized with respect to the sensing interval) $\tau$ follows a negative binomial distribution with probability mass function [17]

$$
P_{\mathrm{NB}}(\tau ; L, \eta)=\left(\begin{array}{c}
\tau+L-1 \\
L-1
\end{array}\right)(1-\eta)^{L} \eta^{\tau},
$$

which is the summation of $L$ geometric random variables with the distribution $G(\eta)$, with $\eta$ denoting the probability that the link is unavailable. When $\tau \leq W$, the coded packet is successfully transmitted through the $r$ th relay path. Thus, the probability of path availability, i.e., of the event $v_{m, r}=1$, is given by

$$
P\left(v_{m, r}=1\right)=P(\tau \leq W)=\sum_{\tau=L}^{W} P_{\mathrm{NB}}(\tau-L ; L, \eta) .
$$

Should the vehicle lack spectrum sensing capability, as it occurs when low-cost circuits are used for communication, the packets immediately collide when the link is occupied by other users. In this case, the erasure probability takes the simplified form

$$
P\left(v_{m, r}=1\right)=(1-\eta)^{L} .
$$

Thus, depending on the communication protocol and on the capability of the vehicles, the erasure probability due to the virtual cell in low-latency vehicular networks with proactive open-loop communication is lower- and upper-bounded by (2) and (3), respectively. If anticipatory mobility management is used, the probability of erasure equals the prediction error probability. Since this is generally low [11], we only consider erasures due to the proactive association within the virtual cell. For ease of exposition, in the balance of this work we assume the same erasure probability of all paths and time instants such that

$$
p_{e}=P\left(v_{m, r}=0\right), \quad \forall m, r .
$$

\section{B. Virtual MIMO Framework}

Let $\mathbf{h}_{m}=\left[h_{m, 1}, \ldots, h_{m, R}\right]^{\top} \in \mathbb{C}^{R}$ be the vector of the fading gains affecting the $R$ paths at time $m$. These are products of the $L$ link fading gains $h_{m, r}=\prod_{l=1}^{L} g_{m, l, r}$. Moreover, let $\mathbf{v}_{m}=\left[v_{m, 1}, \ldots, v_{m, R}\right]^{\top}$ denote the associated erasure vector. The fading erasure channel is defined by the Schur product of $\mathbf{h}_{m}$ and $\mathbf{v}_{m}$ as $\mathbf{h}_{m} \circ \mathbf{v}_{m}=\left[h_{m, 1} v_{m, 1}, \ldots, h_{m, R} v_{m, R}\right]^{\top}$. Here we assume that channel fading gains and erasures are independent. The reason is that the erasures are mainly due to (i) simultaneous access to the same radio resource, (ii) wrong access point association for downlink transmission caused by erroneous anticipatory mobility management, and (iii) deep fading. While the last type of erasures is intrinsically described by the random channel fading gains, (i) and (ii) are independent of channel fading, and hence modeled by the erasure random variables $v_{m, r}$. Then, reliable end-to-end networking 
using the opportunistic links is generated within a virtual MIMO framework [12], where the source node encodes a data packet $\mathbf{x} \in X^{M}$ by using the coding matrix $\tilde{\mathbf{C}}_{m} \in \mathbb{C}^{R \times M}$, and $X$ denotes the signal constellation, i.e., the set of QAM symbols. With $\tilde{\mathbf{C}}_{m} \mathbf{x}$ being transmitted at time instant $m \in\{1, \ldots, M\}$, the received coded packet $y_{m}$ at the destination node is given by [20]

$$
y_{m}=\left(\mathbf{h}_{m} \circ \mathbf{v}_{m}\right)^{\top} \tilde{\mathbf{C}}_{m} \mathbf{x}+\sum_{r=1}^{R} \tilde{n}_{m, r}=\left(\mathbf{h}_{m} \circ \mathbf{v}_{m}\right)^{\top} \tilde{\mathbf{C}}_{m} \mathbf{x}+n_{m},
$$

where $\tilde{n}_{m, r}$ denotes the accumulated additive white Gaussian noise (AWGN) of all links in path $r$ at time $m$. Due to the amplification of the opportunistic link gains, the power spectral density $N_{\mathrm{o}, m}$ of the aggregated AWGN of all paths is time-varying [20].

At the destination node, the received packets are given by the components of vector $\mathbf{y}$, where

$$
\begin{aligned}
\mathbf{y} & =\left[\begin{array}{ccc}
\left(\mathbf{h}_{1} \circ \mathbf{v}_{1}\right)^{\top} & \ldots & \mathbf{0}^{\top} \\
\vdots & \ddots & \vdots \\
\mathbf{0}^{\top} & \ldots & \left(\mathbf{h}_{M} \circ \mathbf{v}_{M}\right)^{\top}
\end{array}\right] \underbrace{\left[\begin{array}{c}
\tilde{\mathbf{C}}_{1} \\
\vdots \\
\tilde{\mathbf{C}}_{M}
\end{array}\right]}_{\mathbf{C}} \underbrace{\left[\begin{array}{c}
n_{1} \\
\vdots \\
n_{M}
\end{array}\right]}_{\mathbf{n}} \\
& =\underbrace{\left[\begin{array}{c}
\left(\mathbf{h}_{1} \circ \mathbf{v}_{1}\right)^{\top} \tilde{\mathbf{C}}_{1} \\
\vdots \\
\left(\mathbf{h}_{M} \circ \mathbf{v}_{M}\right)^{\top} \tilde{\mathbf{C}}_{M}
\end{array}\right]}_{\tilde{\mathbf{H}}_{\mathrm{eq}}(\mathbf{V}, \mathbf{C})} \mathbf{x}+\mathbf{n}=\mathbf{H}_{\mathrm{eq}}(\mathbf{V}, \mathbf{C}) \mathbf{x}+\mathbf{n},
\end{aligned}
$$

and $\mathbf{C}=\left[\begin{array}{lll}\tilde{\mathbf{C}}_{1}^{\top} & \ldots & \tilde{\mathbf{C}}_{M}^{\top}\end{array}\right]^{\top} \in \mathbb{C}^{R M \times M}$ models the cascaded $\tilde{\mathbf{C}}_{m}$. This shows that end-to-end virtual MIMO networking (6) can be analyzed mathematically in the same way as with physical MIMO systems, characterized by the equivalent channel matrix $\tilde{\mathbf{H}}_{\mathrm{eq}}(\mathbf{V}, \mathbf{C}) \in \mathbb{C}^{M \times M}$. This MIMO matrix incorporates the $R M$ Bernoulli random variables modeling the erasures, and can be modified by a suitable choice of the coding scheme [28, p. $350 \mathrm{ff}$.]. Various matrices can be used for coding, e.g., orthonormal matrices and discrete Fourier transform matrices [20], in order to shape the equivalent channel matrix $\tilde{\mathbf{H}}_{\mathrm{eq}}(\mathbf{V}, \mathbf{C})$ for improved error performance.

\section{Conventional PPC}

With conventional end-to-end PPC networking [1], a permutation vector $\mathbf{p}=\left[p_{1}, \ldots, p_{M}\right]$ is defined (see also Fig. 1(a)). Specifically, if the coding scheme assumes that single symbol $x$ is repeatedly transmitted through path $p_{m}$ for $m=1, \ldots, M$, the coding matrix $\tilde{\mathbf{C}}_{m}$ reduces to the vector $\tilde{\mathbf{c}}_{m} \in\{0,1\}^{R}$ whose entries are

$$
\tilde{c}_{m, r}= \begin{cases}1, & \text { if } r=p_{m}, \\ 0, & \text { otherwise. }\end{cases}
$$

Thus, $\tilde{\mathbf{c}}_{m} \in\{0,1\}^{R}$ has only one nonzero entry in the $p_{m}$ th position. The associated erasure channel model yields

$$
\left(\mathbf{h}_{m} \circ \mathbf{v}_{m}\right)^{\top} \tilde{\mathbf{c}}_{m}=\left(\mathbf{h}_{m} \circ \mathbf{v}_{m}\right)^{\top}[\underbrace{0, \ldots, 0}_{p_{m}-1}, 1, \underbrace{0, \ldots, 0}_{R-p_{m}}]^{\top}=h_{m, p_{m}} v_{m, p_{m}} .
$$

TABLE I

GeNeralized PPC PARAMETERs.

\begin{tabular}{|c|c|}
\hline parameter & definition \\
\hline$R$ & number of relay paths \\
\hline$K$ & number of simultaneous accessed paths \\
\hline$M$ & number of used time instants \\
\hline$\widehat{K}$ & number of time repetitions of QAM symbols \\
\hline$\widehat{M}$ & number of path repetitions of QAM symbols \\
\hline$S$ & size of the permutation set \\
\hline$d_{\mathrm{dmin}}$ & minimum Hamming distance of the permutation set \\
\hline
\end{tabular}

As a consequence, the virtual MIMO system model in (6) of the end-to-end PPC networking is transformed into

$$
\mathbf{y}=\left[\begin{array}{c}
h_{1, p_{1}} v_{1, p_{1}} \\
\vdots \\
h_{M, p_{M}} v_{M, p_{M}}
\end{array}\right] x+\mathbf{n}=\left(\mathbf{h}_{\mathbf{p}} \circ \mathbf{v}_{\mathbf{p}}\right) x+\mathbf{n},
$$

where $\mathbf{h}_{\mathbf{p}}$ is defined as $\left[h_{1, p_{1}}, \ldots, h_{M, p_{M}}\right]^{\top} \in \mathbb{C}^{M}$ to represent the fading of the selected paths from the time instant 1 to $M ; \mathbf{v}_{\mathbf{p}}=$ $\left[v_{1, p_{1}}, \ldots, v_{M, p_{M}}\right]^{\top} \in\{0,1\}^{M}$ is defined likewise.

Since conventional PPC only accesses one relay path in the $R$-path route at each time instant, only one QAM symbol is repeatedly transmitted along the time coordinate. Therefore, the PPC throughput is generally lower than other virtual MIMO techniques [1], [20]. Such drawback can be overcome by generalizing the scheme to simultaneously access multiple relay paths (as illustrated in Fig. 1(b)) and including the possibility of transmitting multiple QAM symbols in the packet. This will be elaborated upon in the following section.

\section{Generalization of the End-to-End PPC Networking}

In this section we elaborate on a generalized version of PPCs. This achieves lower error rate, higher throughputs, and stronger robustness to erasures, thus enabling reliable end-toend networking of opportunistic links. We first introduce the encoding scheme of generalized PPC, characterized by one permutation set plus one combination set for simultaneous multipath access. Next, encoding of multiple QAM symbols is addressed. Since the permutation sets previously proposed in [1], [29] are inadequate for the generalized PPC version described here, we develop a two-phase algorithm to generate them as needed. For clarity, the important parameters of the generalized PPC is listed in Table I.

\section{A. Combination and Permutation Sets of the Generalized PPC Technique}

When the PPC is generalized to simultaneously access $K$ paths for $1 \leq K \leq R$ at a certain time instant (as illustrated in Fig.1(b) for $K=2$ ), the indices of the accessed paths can be represented by a $K$-combination vector of a positive-integer set $\{1, \ldots, R\}$. Let $Q_{R, K}$ be a combination set that includes all these possible $K$ combination vectors. For example, $Q_{4,2}$ is given by

$$
Q_{4,2}=\left\{\left[\begin{array}{l}
1 \\
2
\end{array}\right],\left[\begin{array}{l}
1 \\
3
\end{array}\right],\left[\begin{array}{l}
1 \\
4
\end{array}\right],\left[\begin{array}{l}
2 \\
3
\end{array}\right],\left[\begin{array}{l}
2 \\
4
\end{array}\right],\left[\begin{array}{l}
3 \\
4
\end{array}\right]\right\},
$$

where the entries in the combination vector are the indices of the paths accessed by the generalized PPC. The data are 
encoded by the permutation vector which describes, rather than the path directly, the path combination at each time instant. For instance, with $(R, K)=(4,2)$ the permutation vector $\mathbf{p}=[2,3]^{\top}$ describes the combinations $[1,3]^{\top}$ and $[1,4]^{\top}$ in $Q_{4,2}$ (10). This means that the end-to-end network using generalized PPC accesses the first and third paths at the first time instant, and the first and fourth paths at the second time instant.

To select $M$ combination vectors from $Q_{R, K}$, we define the $M$-permutation vectors ${ }^{1} \mathbf{p}$ as a vector of a positive integer set $\left\{1, \ldots,\left(\begin{array}{l}R \\ K\end{array}\right)\right\}$, which is used to describe the simultaneous access of $K$ out of the $R$ relay paths at each time instant. Specifically, to specify a generalized PPC, a permutation set $\mathcal{P}_{M, R, K}\left(S, d_{\min }\right)$ is required which comprises $S M$ permutation vectors, where

$$
S \leq \frac{\left(\begin{array}{l}
R \\
K
\end{array}\right) !}{\left(\left(\begin{array}{l}
R \\
K
\end{array}\right)-M\right) !}=\left(\begin{array}{l}
R \\
K
\end{array}\right) \times\left(\left(\begin{array}{l}
R \\
K
\end{array}\right)-1\right) \times \cdots \times\left(\left(\begin{array}{l}
R \\
K
\end{array}\right)-M+1\right),
$$

The number of bits encoded by the permutations is $\left\lfloor\log _{2} S\right\rfloor$, with $\lfloor\cdot\rfloor$ the floor operator. The minimum Hamming distance $d_{\text {min }}$ of this set is defined as

$$
d_{\min }=\min _{\forall \mathbf{p}_{i}, \mathbf{p}_{j} \in \mathcal{P}_{M, R, K}\left(S, d_{\min }\right)} d\left(\mathbf{p}_{i}, \mathbf{p}_{j}\right),
$$

where $d\left(\mathbf{p}_{i}, \mathbf{p}_{j}\right)$ is the Hamming distance between the permutation vectors $\mathbf{p}_{i}$ and $\mathbf{p}_{j}$. As will be expounded in next section, both diversity and error floor of the end-to-end generalized PPC networking depend on the value of $d_{\min }$.

The first advantage of accessing multiple paths is throughput increase. Taking $(M, R)=(3,5)$ as an example, and comparing the cases with $K=1$ and $K=2$, the maximum value of $S$ increases from 60 to 720 , implying that the bits conveyed by the permutation vectors are almost doubled (from 5 bits to 9 bits). Such throughput improvement can be easily translated into a reliability enhancement by increasing the minimum Hamming distance $d_{\min }$ rather than the size $S$. Thus, accessing multiple paths provides a trade-off between throughput and reliability which is more flexible and better than accessing a single path at a time as in conventional PPC. Moreover, the generalized PPC is more robust to transmission outages (erasures), because when $K$ paths are exploited at the same time the likelihood that the destination fails to receive any information at a certain time instant decreases exponentially as the value of $K$ increases. We shall expand on this later, from the perspective of diversity loss and error floor.

\section{B. Encoding of Generalized PPC}

With generalized PPC, the encoder operates as follows: First, given the parameters $(M, R, K)$, the permutation set $\mathcal{P}_{M, R, K}\left(S, d_{\min }\right)$ is designed off-line and made known to both source and destination node. The binary information data select the QAM symbol and a permutation vector $\mathbf{p} \in$

\footnotetext{
${ }^{1}$ This vector is sometimes defined as partial permutation vector, since only some of the integers $\left\{1, \ldots,\left(\begin{array}{l}R \\ K\end{array}\right)\right\}$ appear among the vector components.
}

$\mathcal{P}_{M, R, K}\left(S, d_{\min }\right)$. Then, the encoder forms a combination matrix $\mathbf{Q}(\mathbf{p}) \in \mathbb{N}^{M \times K}$ whose transpose of the $m$ th row vector $\mathbf{q}_{m}=\left[q_{m, 1}, \ldots, q_{m, K}\right]^{\top}$ is the $p_{m}$ th combination vector in the combination set $Q_{R, K}$. The source node sequentially accesses the multiple relay paths according to this combination matrix, which leads to the input-output relation

$$
\mathbf{y}=\left[\begin{array}{c}
\left(\mathbf{h}_{\mathbf{q}_{1}} \circ \mathbf{v}_{\mathbf{q}_{1}}\right)^{\top} \\
\vdots \\
\left(\mathbf{h}_{\mathbf{q}_{M}} \circ \mathbf{v}_{\mathbf{q}_{M}}\right)^{\top}
\end{array}\right] \mathbf{x}+\mathbf{n}=\left(\mathbf{H}_{\mathbf{Q}} \circ \mathbf{V}_{\mathbf{Q}}\right) \mathbf{x}+\mathbf{n},
$$

where $\mathbf{x}=[x, \ldots, x]^{\top}$ denotes $K$ repetitions of QAM symbol $x$. Let $\mathbf{h}_{\mathbf{q}_{m}}=\left[h_{m, q_{m, 1}}, \ldots, h_{m, q_{m, K}}\right]^{\top}$ and $\mathbf{v}_{\mathbf{q}_{m}}=\left[v_{m, q_{m, 1}}, \ldots, v_{m, q_{m, K}}\right]^{\top}$ model the channel fading gains and the erasures of the $K$ paths accessed at time $m$, respectively, with $h_{m, q_{m, k}}$ and $v_{m, q_{m, k}}$ corresponding to the $q_{m, k}$ th relay path at time $m$. Compared with (9), the equivalent channel matrix becomes the Schur product of $\mathbf{H}_{\mathbf{Q}} \in \mathbb{C}^{M \times K}$ and $\mathbf{V}_{\mathbf{Q}} \in\{0,1\}^{M \times K}$, whose transpose of the $m$ th row vectors are $\mathbf{h}_{\mathbf{q}_{m}}$ and $\mathbf{v}_{\mathbf{q}_{m}}$, respectively.

A further generalization shows that, like with the tradeoff between $S$ and $d_{\min }$, the throughput of the end-to-end PPC networking can be elevated by increasing the number of different transmitted QAM symbols in a PPC packet. In particular, let $(\widehat{M}, \widehat{K})$ indicate that the same QAM symbol is repeatedly transmitted at $\widehat{M}$ successive time instants using $\widehat{K}$ paths. The transmitted QAM matrix $\mathbf{X} \in \mathcal{X}^{M \times K}$ is represented as

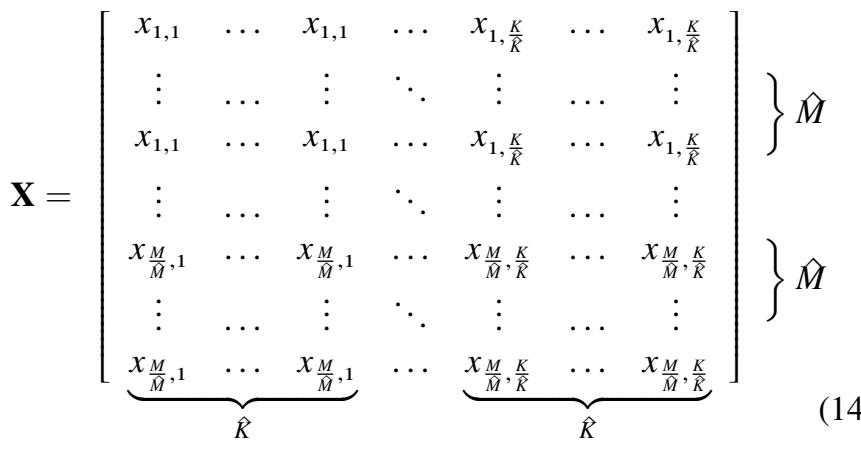

Thus, the transmission model at the $m$ th time instant is given by

$$
y_{m}=\left(\mathbf{h}_{\mathbf{q}_{m}} \circ \mathbf{v}_{\mathbf{q}_{m}}\right)^{\top} \mathbf{x}_{m}+n_{m}, \quad m=1, \ldots, M,
$$

where $\mathbf{x}_{m}$ is the transpose of the $m$ th row vector of $\mathbf{X}$, representing the transmitted QAM vector that comprise $\frac{K}{\widehat{K}}$ different QAM symbols.

For the entire PPC packet, in addition to the permutation vector $\mathbf{p}$, we have $\frac{M K}{\grave{M} \hat{K}}$ QAM symbols distributed in the timepath plane. The throughput is inversely proportional to the number of repetitions $\hat{M} \hat{K}$, i.e.,

$$
\text { throughput }=\frac{\log _{2}\lfloor S\rfloor+\frac{M K}{\widehat{M} \log _{2}}|X|}{M},
$$

where these transmission parameters, e.g., modulation schemes and number of repetitions, are selected according to the channel conditions and the system requirements. It can be seen that the design space of generalized PPC is significantly wider than that of conventional PPC. Instead of conducting extensive numerical simulations, in next section 
we theoretically analyze the error rate, which reveals how, given a certain throughput requirement, one should increase the value of $\hat{K}$ from 1 to $K$ as erasure probabilities increase from low to high. The diversity induced by QAM symbols and permutation vectors are $\widehat{M}$ and $d_{\min }$, respectively. Theoretical analysis shows that repetition in the time coordinate allows a trade-off between error rate and throughput.

\section{Permutation Set Generation}

The simultaneous access of multiple paths increases the number of usable permutation vectors. For example, with $(R, K)=(5,2)$, generalized PPC requires a permutation vector constructed from the positive integer set $\{1, \ldots, 10\}$. The works [1], [29] exhibit only permutations with entries ranging from 1 to 6 , thus new permutation sets need be designed here.

Generating permutation sets is a complicated optimization problem. Considering a toy example with $(M, R, K)=$ $(4,4,2)$, we see from $(11)$ that the number of usable permutation vectors is 360 . If we want to load 4 bits on the permutation vector, then $S=16$, and an exhaustive search has to identify the optimal permutation set from $\left(\begin{array}{c}360 \\ 16\end{array}\right) \approx 2.7 \cdot 10^{27}$ permutation sets by comparing the Hamming distances among the vectors in the set for hierarchical optimization [30]. First, the minimum Hamming distance $d_{\min }$ should be maximized. Next, since the error rate is not only dominated by $d_{\min }$ but also by the number of permutation pairs with Hamming distance equal to $d_{\min }$, for those permutation sets with the same $d_{\min }$, the one with the smallest number of minimum Hamming distances $d\left(\mathbf{p}_{i}, \mathbf{p}_{j}\right)$ for all $(i, j)$ should be selected.

With more relay paths or more bits loaded on the permutation vector, the complexity becomes quickly prohibitive: for example, with $(M, R, K)=(4,6,2)$ and 6 bits conveyed by the permutation vector, the exhaustive search needs to compare around $7.1 \cdot 10^{199}$ permutation sets with size $S=64$. Even worse, when the minimum Hamming distance is given, the maximal value of $S$ is unknown due to the constraint of $d_{\min }$. Size maximization is related to finding a maximum clique in a graph whose vertices are all permutation vectors, and whose edges join permutation pairs with a distance larger than or equal to $d_{\min }$. Exhaustive search requires $O\left(V^{S_{\max }}\right)$

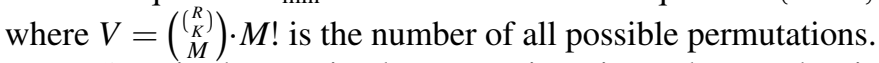
Here, $S_{\max }$ is the maximal permutation size, whose value is unknown before exhaustive search. Its theoretical upper bound is investigated in [31]. Although this optimization can be solved off-line, such that its complexity is less significant, we propose a simple and effective two-phase greedy algorithm for such optimization. The algorithm first generates all the usable permutation vectors in a candidate pool. Then, the permutation vector is sequentially moved from the pool to a new set. When one permutation is moved, the remaining permutations with Hamming distances less than the specified $d_{\min }$ are removed from the pool. By using the criterion that minimizes the number of removed permutations in the candidate pool, such greedy algorithm iteratively constructs a permutation set with the largest value of $S$ and guaranteed $d_{\min }$. After the candidate pool is emptied, we have a permutation set with desired $d_{\min }$, but its size is generally not a power of 2, or larger than required. The second phase consists of shrinking the permutation set by sequentially removing the $i$ th permutation vector with largest number of $d\left(\mathbf{p}_{i}, \mathbf{p}_{j}\right)=d_{\text {min }}$ for all $j$. This procedure is repeated until the size becomes $S$, a power of 2. Note that for small $S$, we may remove all the vectors with Hamming distance equal to $d_{\min }$. In this case, we can increase $d_{\min }$ for better reliability. Most of the complexity of this greedy algorithm is caused by the identification of the permutation pairs whose Hamming distance is larger than or equal to $d_{\min }$, which is $O\left(V^{2}\right)$. Since we have large values of $V$ and $S_{\max } \gg 2$, the complexity is shown to be much lower than that of the exhaustive search, i.e., $O\left(V^{S_{\max }}\right)$. Numerical simulations, described later, support our conjecture that the proposed two-phase greedy algorithm can yield a satisfactory performance of generalized end-to-end PPC networking.

\section{Theoretical Error-Rate Analysis and Design EXPLORATION}

To shed light on the benefits of generalizing PPC with the inclusion of multiple QAM symbols in the packet, and transmit them through multiple relay paths whose indices are also part of the data packet, we comprehensively analyze the performance, including the error rate, diversity, and error floor. With an error-rate model, the design space of generalized PPC can be explored faster and in a systematic way, which provides design guidelines that optimize the generalized PPC transmission parameters and achieve efficient end-to-end networking of opportunistic links.

\section{A. Error Rate Analysis}

For generalized PPC networking, the data are loaded on a QAM matrix $\mathbf{X}$ and a permutation vector $\mathbf{p}$. The PEP $f(\boldsymbol{\pi} \rightarrow \tilde{\boldsymbol{\pi}})$ is the conditional probability that, when the packet $\pi \triangleq(\mathbf{X}, \mathbf{p})$ is transmitted, the competing packet $\tilde{\boldsymbol{\pi}}=(\tilde{\mathbf{X}}, \tilde{\mathbf{p}})$ has a higher likelihood:

$$
f(\boldsymbol{\pi} \rightarrow \tilde{\boldsymbol{\pi}}) \triangleq P(\Lambda(\boldsymbol{\pi}, \tilde{\boldsymbol{\pi}})<0),
$$

where $\Lambda(\boldsymbol{\pi}, \tilde{\boldsymbol{\pi}})$ is the log-likelihood ratio (LLR)

$$
\Lambda(\boldsymbol{\pi}, \tilde{\boldsymbol{\pi}}) \triangleq \log (P(\mathbf{y} \mid \boldsymbol{\pi}, \mathbf{H}, \mathbf{V}) / P(\mathbf{y} \mid \tilde{\boldsymbol{\pi}}, \mathbf{H}, \mathbf{V})) .
$$

Since $n_{m}$ is AWGN with noise power spectral density $N_{\mathrm{o}, m}$, we obtain

$$
P(\mathbf{y} \mid \boldsymbol{\pi}, \mathbf{H}, \mathbf{V})=\pi^{-M} \prod_{m=1}^{M} N_{0, m}^{-1} e^{\frac{1}{N_{0, m}}\left|y_{m}-\left(\mathbf{h}_{\mathbf{q}_{m}} \circ \mathbf{v}_{\mathbf{q}_{m}}\right)^{\top} \mathbf{x}_{m}\right|^{2}} .
$$

By using (18) and (19), the LLR is formulated as

$$
\begin{aligned}
\Lambda(\mathbf{X}, \mathbf{p}, \tilde{\mathbf{X}}, \tilde{\mathbf{p}}) & =\sum_{m=1}^{M} \frac{1}{N_{\mathrm{o}, m}}\left(\mid\left(\mathbf{h}_{\mathbf{q}_{m}} \circ \mathbf{v}_{\mathbf{q}_{m}}{ }^{\top} \mathbf{x}_{m}-\left.\left(\mathbf{h}_{\tilde{\mathbf{q}}_{m}} \circ \mathbf{v}_{\tilde{\mathbf{q}}_{m}}\right)^{\top} \tilde{\mathbf{x}}_{m}\right|^{2}\right.\right. \\
& \left.-2 \operatorname{Re}\left\{\left(\left(\mathbf{h}_{\mathbf{q}_{m}} \circ \mathbf{v}_{\mathbf{q}_{m}}\right)^{\top} \mathbf{x}_{m}-\left(\mathbf{h}_{\tilde{\mathbf{q}}_{m}} \circ \mathbf{v}_{\tilde{\mathbf{q}}_{m}}\right)^{\top} \tilde{\mathbf{x}}_{m}\right)^{*} n_{m}\right\}\right),
\end{aligned}
$$

where $\operatorname{Re}\{\cdot\}$ denotes real part, and $(\cdot)^{*}$ complex conjugate. For the erroneously-detected signals at the $m$ th time instant, we define the associated combination vector and QAM vector 
as $\tilde{\mathbf{q}}_{m}$ and $\tilde{\mathbf{x}}_{m}$, respectively. Given that $n_{m}$ is a Gaussian random variable, and conditioned on the fading gains and erasures $(\mathbf{H}, \mathbf{V}), \Lambda(\boldsymbol{\pi}, \tilde{\boldsymbol{\pi}})$ is also a conditionally Gaussian random variable with mean

$$
\bar{\Lambda}=\sum_{m=1}^{M} \frac{1}{N_{0, m}} \mu_{m},
$$

where

$$
\mu_{m} \triangleq\left|\left(\mathbf{h}_{\mathbf{q}_{m}} \circ \mathbf{v}_{\mathbf{q}_{m}}\right)^{\top} \mathbf{x}_{m}-\left(\mathbf{h}_{\tilde{\mathbf{q}}_{m}} \circ \mathbf{v}_{\tilde{\mathbf{q}}_{m}}\right)^{\top} \tilde{\mathbf{x}}_{m}\right|^{2} .
$$

Likewise, its variance $\sigma_{\Lambda}^{2}$ is

$$
\sigma_{\Lambda}^{2}=2 \mu_{m}=\sum_{m=1}^{M} \frac{2}{N_{0, m}} \mu_{m} .
$$

The conditional moment-generating function (MGF) of $\Lambda$ conditioned on $(\mathbf{H}, \mathbf{V})$ is derived as

$$
M_{\Lambda}(s \mid \mathbf{H}, \mathbf{V})=e^{\left(s+s^{2}\right) \bar{\Lambda}} .
$$

To remove the condition of fading erasure channels $(\mathbf{H}, \mathbf{V})$, we can marginalize and rewrite (24)

$$
\begin{aligned}
& M_{\Lambda}(s)= E_{\mathbf{H}, \mathbf{V}}\left\{M_{\Lambda}(s \mid \mathbf{H}, \mathbf{V})\right\}=E_{\mathbf{H}, \mathbf{V}}\left\{e^{\left(s+s^{2}\right) \bar{\Lambda}}\right\} \\
& \stackrel{(a)}{=} \prod_{m=1}^{M} E_{\mu_{m}}\left\{e^{\frac{s+s^{2}}{N_{0, m}} \mu_{m}}\right\}=\prod_{m=1}^{M} M_{\mu_{m}}\left(\frac{s+s^{2}}{N_{\mathrm{O}, m}}\right),
\end{aligned}
$$

where $(a)$ is due to the fact that $\mu_{m}$ in (22) can be treated as a random variable including random fading gains and erasures. The computation of the unconditional MGF $M_{\Lambda}(s)$ thus is reformulated as calculating the product of $M_{\mu_{m}}\left(\frac{s+s^{2}}{N_{0, m}}\right)$ for $m=1, \ldots, M$. The following lemma shows the derivation of $M_{\mu_{m}}\left(\frac{s+s^{2}}{N_{0, m}}\right)$, where for simplicity's sake we reduce ourselves to the case of a single transmitted QAM symbol, i.e., the first generalization model in (13), which allows us to omit the subscripts of $x$ and $\tilde{x}$.

Lemma 1: The unconditional MGF $M_{\mu_{m}}\left(\frac{s+s^{2}}{N_{0, m}}\right)$ is derived as follow:

$$
\begin{aligned}
& M_{\mu_{m}}\left(\frac{s+s^{2}}{N_{0}}\right)=\sum_{i=1}^{3} G_{L, 1}^{1, L}\left[-\frac{\left(s+s^{2}\right) \alpha_{i}}{N_{0}} \mid \begin{array}{cc}
0 & \ldots \\
0
\end{array}\right] P_{\mu_{m}^{(i)}}
\end{aligned}
$$

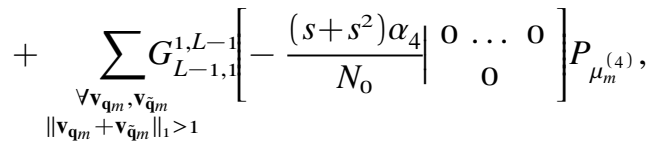

where the L1-norm $\|\cdot\|_{1}$ denotes the number of nonzero terms in its argument. The equation (26) is computed by first averaging the channel fading conditioned on $\left(\mathbf{v}_{\mathbf{q}_{m}}, \mathbf{v}_{\tilde{q}_{m}}\right)$, which can be categorized into four cases depending on different erasure realizations. The results are expressed by using the parameter $\alpha_{i}$, the probability $P_{\mu_{m}^{(i)}}$ of the $i$ th category, and the Meijer G-function:

$$
\begin{aligned}
& G_{t, u}^{w, v}\left[x \mid \begin{array}{lll}
a_{1} & \ldots & a_{t} \\
b_{1} & \ldots & b_{u}
\end{array}\right] \\
& \triangleq \frac{1}{2 \pi j} \int_{-j \infty}^{j \infty} \frac{\prod_{i=1}^{w} \Gamma\left(b_{i}+s\right) \prod_{i=1}^{v} \Gamma\left(1-a_{i}-s\right)}{\prod_{i=m+1}^{u} \Gamma\left(1-b_{i}-s\right) \prod_{i=n+1}^{t} \Gamma\left(a_{i}+s\right)} x^{s} \mathrm{~d} s,
\end{aligned}
$$

where $w \leq u, v \leq t$, and all these four parameters are positive integers. For the first two cases, we have $\mathbf{v}_{\tilde{\mathbf{q}}_{m}}=\mathbf{0}$ and one nonzero entry of $\mathbf{v}_{\mathbf{q}_{m}}$, or inversely $\mathbf{v}_{\mathbf{q}_{m}}$ and one nonzero entry of $\mathbf{v}_{\tilde{\mathbf{q}}_{m}}=\mathbf{0}$. In the third case, we have both one nonzero entry of $v_{q_{m, k}}$ and $v_{\tilde{q}_{m, k^{\prime}}}$ with the condition $q_{m, k}=\tilde{q}_{m, k^{\prime}}$, i.e., both $x$ and $\tilde{x}$ are propagated through the same available path. Last, the fourth case comprises the realizations of $\left\|\mathbf{v}_{\mathbf{q}_{m}}+\mathbf{v}_{\tilde{\mathbf{q}}_{m}}\right\|_{1}>1$, meaning that at least two paths are available for $x$ and $\tilde{x}$. The associated parameters $\alpha_{i}$ of these four cases are given by

$$
\begin{aligned}
& \alpha_{1}=|x|^{2}, \quad \alpha_{2}=|\tilde{x}|^{2}, \quad \alpha_{3}=|x-\tilde{x}|^{2}, \\
& \alpha_{4}=Z_{m}^{(v)}|x-\tilde{x}|^{2}+\left(\left\|\mathbf{v}_{\mathbf{q}_{m}}\right\|_{1}-Z_{m}^{(v)}\right)|x|^{2}+\left(\left\|\mathbf{v}_{\tilde{\mathbf{q}}_{m}}\right\|_{1}-Z_{m}^{(v)}\right)|\tilde{x}|^{2},
\end{aligned}
$$

where $Z_{m}$ denotes the number of total paths that are jointly accessed by $x$ and $\tilde{x}$ at time $m$. For example, if $\mathbf{q}_{m}=[1,2,3]^{\top}$ and $\tilde{\mathbf{q}}_{m}=[2,3,4]^{\top}$, we have $Z_{m}=2$ since the second and third paths are jointly accessed by $x$ and $\tilde{x}$. Analogously , $Z_{m}^{(v)} \leq Z_{m}$ denotes the number of total available paths jointly accessed by both $x$ and $\tilde{x}$. Following the previous example with $\left(v_{m, 2}, v_{m, 3}\right)=(0,1)$, we have $Z_{m}^{(v)}=1$ since only the third path is available and jointly accessed by $x$ and $\tilde{x}$.

Using the parameters defined above, the probabilities of these four cases used in (26) are

$$
P_{\mu_{m}^{(i)}}=\left\{\begin{array}{cc}
\left(K-Z_{m}\right) \cdot p_{e}^{2 K-Z_{m}-1}\left(1-p_{e}\right), & i=1,2 \\
Z_{m} \cdot p_{e}^{2 K-Z_{m}-1}\left(1-p_{e}\right), & i=3, \\
p_{e}^{2 K-Z_{m}-\left\|\mathbf{v}_{\mathbf{q}_{m}}+\mathbf{v}_{\tilde{\mathbf{q}}_{m}}\right\|_{1}}\left(1-p_{e}\right)^{\left\|\mathbf{v}_{\mathbf{q}_{m}}+\mathbf{v}_{\tilde{\mathbf{q}} m}\right\|_{1},}, & i=4 .
\end{array}\right.
$$

From this equation and (28), only $\alpha_{4}$ and $P_{\mu_{m}^{(4)}}$ of the fourth case depend on the realization of $\mathbf{v}_{\mathbf{q}_{m}}$ and $\mathbf{v}_{\tilde{\mathbf{q}}_{m}}$ and that is the reason of having the second summation at the right hand side (RHS) in (26), which is used to consider various realizations of erasures that satisfying the conditions of $\left\|\mathbf{v}_{\mathbf{q}_{m}}+\mathbf{v}_{\tilde{\mathbf{q}}_{m}}\right\|_{1}>1$. The details of the derivations are provided in Appendix A.

With $M_{\Lambda}(s)$ at hand, the PEP is computed by integration [32]

$$
f(\boldsymbol{\pi} \rightarrow \tilde{\boldsymbol{\pi}})=\frac{1}{2 \pi j} \int_{\hat{s}-j \infty}^{\hat{s}+j \infty} M_{\Lambda}(s) \frac{\mathrm{d} s}{s},
$$

where the Gaussian approximation, saddlepoint approximation [33], or the Gauss-Chebyshev quadratures can be adopted [34] to tightly approximate this integral. Then, the BER $P_{\mathrm{b}}$ is derived by applying the union bound [35]

$$
P_{\mathrm{b}} \leq \frac{1}{|X| \frac{M K}{M K} S\left(\frac{M K}{\bar{M} \hat{K}} \log _{2}|X|+\log _{2} S\right)} \sum_{\forall \boldsymbol{\pi}} \sum_{\forall \tilde{\boldsymbol{\pi}}} d_{\boldsymbol{\pi}, \tilde{\boldsymbol{\pi}}}^{(b)} f(\boldsymbol{\pi} \rightarrow \tilde{\boldsymbol{\pi}}),
$$

where $|X|$ denotes the cardinality of $\mathcal{X}$, and $d_{\boldsymbol{\pi} \tilde{\boldsymbol{\pi}}}^{(b)}$ the Hamming distance between the demapped bits of transmitted and erroneously detected data. In summary, our analysis reveals the connections among error rate performance, transmission parameters, and the environment parameters. For example, the number of cascaded links $L$ is represented in the Meijer G-function used to described the moment generating function. For larger $L$, the performance deteriorates as the amount of fading increases [36]. The parameters $\alpha_{3}$ and $\alpha_{4}$ in (28) are functions of $|x-\tilde{x}|$ : thus, the higher the modulation scheme, the smaller $\alpha_{3}$ and $\alpha_{4}$, leading to worse error performance. 
Additionally, the erasure probability $p_{e}$ is used to describe the probabilities of different erasure realizations, which affects the error rate performance as shown in (26) and (29).

\section{B. Diversity Analysis}

Determining, at least for high SNR, the slope of the curve of error rate vs. SNR, the diversity is reflected in the SNR exponent. This can be analyzed by applying the Chernoff bound with $s=\hat{s}=\frac{1}{2}$, the value yielding $\frac{d \log M_{\Lambda}(s)}{d s}=0$ :

$$
f_{\mathrm{CB}}(\boldsymbol{\pi} \rightarrow \tilde{\boldsymbol{\pi}})=M_{\Lambda}(\hat{\boldsymbol{s}})=\prod_{m=1}^{M} M_{\mu_{m}}\left(\frac{-1}{4 N_{\mathrm{o}, m}}\right) .
$$

We can see that the diversity of the generalized PPC technique can be up to $M$ when all $M_{\mu_{m}}\left(\frac{-1}{4 N_{0, m}}\right)$ are functions of the SNR. Nevertheless, we have up to $M-d_{\min }$ time instants where $\mathbf{q}_{m}=$ $\tilde{\mathbf{q}}_{m}$ because of $p_{m}=\tilde{p}_{m}$ at these time instants. At these time instants, only the last two cases in (26) exist, since the first and second cases occur only if $\mathbf{q}_{m} \neq \tilde{\mathbf{q}}_{m}$. Moreover, for the fourth case, due to the fact that $\left\|\mathbf{v}_{\mathbf{q}_{m}}\right\|_{1}=\left\|\mathbf{v}_{\tilde{\mathbf{q}}_{m}}\right\|_{1}=Z_{m}^{(v)}$, the last two terms of $\alpha_{4}$ in (28) disappear, and hence $\alpha_{4}$ is reduced to

$$
\alpha_{4}=Z_{m}^{(v)}|x-\tilde{x}|^{2},
$$

which becomes zero when $x=\tilde{x}$. Likewise, $\alpha_{1}=0$ (28) if $x=\tilde{x}$. Thus, we discover that for the $m$ th time instant with $p_{m}=\tilde{p}_{m}$ and $x=\tilde{x}$, both $\alpha_{1}$ and $\alpha_{4}$ are equal to 0 , leading to

$$
M_{\mu_{m}}\left(\frac{-1}{4 N_{\mathrm{o}, m}}\right)=1, \quad p_{m}=\tilde{p}_{m}, x=\tilde{x},
$$

which is independent of SNR: thus, diversity is lost. In other words, the diversity of the permutation vector is dominated by the minimum Hamming distance $d_{\min }$ of the permutation set, regardless of the number of accessed paths $K$. Nevertheless, accessing multiple paths still enjoys several advantages such as higher throughput as mentioned previously. The error floor is also reduced, as we shall show later.

For QAM diversity, although the result in Lemma 1 is based on a model with a single QAM symbol transmission, from the derivation of the MGF $M_{\mu_{m}}$ and (28) we can see that the MGF $M_{\mu_{m}}\left(\frac{-1}{4 N_{0, m}}\right)$ is a function of SNR as long as $x_{m, k} \neq \tilde{x}_{m, k}$ for any $m$ and $k$. When $\widehat{M}$ time repetitions are used, the QAM diversity equals $\widehat{M}$, since at the remaining time instants we may have identical $x_{m^{\prime}, k}$ and $\tilde{x}_{m^{\prime}, k}$.

The maximal diversity induced by QAM symbols and by permutation vectors is determined by the number of time repetitions $\hat{M}$ and by the minimum Hamming distance $d_{\text {min }}$, respectively. In practice, diversity may be lower when random erasures are introduced. Therefore, a good design rule is to set $\widehat{M} \geq d_{\min }$ and $\widehat{K}=1$, which yields a satisfactory error rate performance with high throughput. In this case, the diversity is lower bounded by $d_{\min }$, while the throughput is maximized as $\log _{2}\lfloor S\rfloor+K \frac{M}{\bar{M}} \log _{2}|X|$. To further improve the error rate performance, the designer may also split the QAM symbol into multiple points with lower modulations, and transmit at different relay paths. These design guideline is useful for low erasure probability. When erasures occurs frequently, error floor considerations should be added, and hence different design guidelines should be considered.

\section{Error Floor Analysis}

Due to erasures, an error floor appears for end-to-end PPC networking at high SNR. Taking $K=1$ and the permutation vector $\mathbf{p}=[4,3,2,1]$ as an example, when the erasures occur at the last two time instants, the destination node cannot distinguish $\mathbf{p}$ from $\tilde{\mathbf{p}}=[4,3,1,2]$ even for infinitely large SNR. Thus, for high erasure probabilities the PPC parameters should be carefully designed to minimize such error rate saturation. We derive the error floor of the generalized PPC in the following lemma.

Lemma 2: The error floor of the generalized end-to-end PPC networking is lower bounded by

$$
P_{\mathrm{b}, N_{0, m} \rightarrow 0} \geq \frac{\frac{M K}{\widehat{M} \hat{K}} \log _{2}|X| \cdot p_{e}^{\widehat{M} \hat{K}}+\log _{2}\lfloor S\rfloor \cdot p_{e}^{2 d_{\min }}}{2\left(\frac{M K}{\widehat{M} \hat{K}} \log _{2}|X|+\log _{2}\lfloor S\rfloor\right)} .
$$

The details of the derivations are provided in Appendix B.

Inequality (35) is the weighted summation of the error floors of the data conveyed by QAM symbols and permutation vectors, respectively. The former is exponentially proportional to $\hat{K} \hat{M}$, the total QAM repetition in the time-path plane. The latter is exponentially proportional to the minimum Hamming distance. When a higher-order modulation scheme is used, the error floor is closer to $p_{e}^{\widehat{M} \hat{K}}$ because more bits are conveyed by each QAM symbol. In fact, the error floor of the permutation vector is exponentially proportional to $2\left(K-Z_{m}\right)$ for the time instant $m$ with $p_{m} \neq \tilde{p}_{m}$, then lower bounded by $2 d_{\min }$ as derived in Appendix B. This means that although the diversity of the permutation vector is independent of $K$, its error floor is actually reduced by having $K \geq 1$.

\section{Rotation Technique for Generalized End-to-End PPC NETWORKING}

In this section, we describe a phase-rotation technique aimed at increasing the diversity gain and reducing the probability of diversity loss and the error floor. For the diversity achieved through the permutation vector, which was theoretically derived in previous Section, we provide now an intuitive explanation: As shown in (22), we lose diversity when

$$
\mu_{m}=0, \quad p_{m}=\tilde{p}_{m}, \mathbf{x}_{m}=\tilde{\mathbf{x}} .
$$

Therefore, the diversity introduced by the permutation vector equals the number of nonzero $\mu_{m}$, which is the Hamming distance between $\mathbf{p}$ and $\tilde{\mathbf{p}}$, that is, the number of time instants where $p_{m} \neq \tilde{p}_{m}$. The phase-rotation technique we are going to describe yields a nonzero $\mu_{m}$ even at time instants with $p_{m}=$ $\tilde{p}_{m}$, and hence increases diversity. Specifically, we rotate the phase of transmitted QAM symbols by an angle $\theta(\mathbf{p})$. Since the QAM symbols are uniformly distributed on the four-quadrant grid, the rotation angle should be less than $\pi / 2$ to avoid ambiguities:

$$
\theta\left(\mathbf{p}_{s}\right)=\frac{(s-1) \pi}{2 S}, \quad s=1, \ldots, S .
$$


The generalized end-to-end PPC networking model in (15) becomes

$$
y_{m}=\left(\mathbf{h}_{\mathbf{q}_{m}} \circ \mathbf{v}_{\mathbf{q}_{m}}\right)^{\top} \mathbf{x}_{m} e^{\theta(\mathbf{p})}+n_{m}, \quad m=1, \ldots, M .
$$

Compared with (36), even when $p_{m}=\tilde{p}_{m}$ and $\mathbf{x}_{m}=\tilde{\mathbf{x}}_{m}$, we still have nonvanishing $\mu_{m}$ :

$$
\mu_{m}=\left|\left(\mathbf{h}_{\mathbf{q}_{m}} \circ \mathbf{v}_{\mathbf{q}_{m}}\right)^{\top} \mathbf{x}_{m}\right|^{2}\left|e^{\theta(\mathbf{p})}-e^{\theta(\tilde{\mathbf{p}})}\right|^{2}, \quad p_{m}=\tilde{p}_{m}, \mathbf{x}_{m}=\tilde{\mathbf{x}}_{m},
$$

and thus the diversity induced by the permutation increases from $d_{\min }$ to $M$. From (39) we can see that uniform partitions of the angles in (37) can maximize the minimum distance between the PPC codewords associated to an arbitrary pair of permutation vectors.

Now, even when we have nonzero $\mu_{m}$ for all the $M$ time instants, they may be nulled when erasures occur on the all the accessed paths, thus leading to a diversity loss. The probability of diversity loss $P\left(\mu_{m}=0\right)$ increases when certain paths are used jointly to propagate $\mathbf{x}_{m}$ and $\tilde{\mathbf{x}}_{m}$. Taking the example of $\left(p_{m}, \tilde{p}_{m}\right)=(1,2), R=4$, and the combination vector set $Q_{4,2}$ in (10), the associated relay paths are $\mathbf{q}_{m}=[1,2]^{\top}$ and $\tilde{\mathbf{q}}_{m}=[1,3]^{\top}$, respectively, In this case, we have $Z_{m}=1$. Assuming that a single QAM symbol is transmitted in this PPC codeword, the value of $\mu_{m}$ in (22) can be computed as

$$
\mu_{m}=\left|h_{2} v_{2}-h_{3} v_{3}\right|^{2}|x|^{2}, \quad P\left(\mu_{m}=0\right)=p_{e}^{2},
$$

where $P\left(\mu_{m}=0\right)$ depends on the number of erasures in $\mu_{m}$. Meanwhile, for the case of $\left(p_{m}, \tilde{p}_{m}\right)=(1,6)$, we have $\tilde{\mathbf{q}}=[3,4]^{\top}$, so that $Z_{m}=0$ and

$$
\mu_{m}=\left|h_{1} v_{1}+h_{2} v_{2}-h_{3} v_{3}-h_{4} v_{4}\right|^{2}|x|^{2}, \quad P\left(\mu_{m}=0\right)=p_{e}^{4},
$$

leading to a smaller value of $P\left(\mu_{m}=0\right)$. This implies that a diversity loss is less likely when $\mathbf{x}_{m}$ and $\tilde{\mathbf{x}}_{m}$ are transmitted through entirely different paths, i.e., $Z_{m}=0$. The rotation technique can also reduce the diversity loss probability when $Z_{m} \neq 0$. With rotations, (40) can be reformulated as

$$
\mu_{m}=\left|h_{1} v_{1}\left(e^{\theta(\mathbf{p})}-e^{\theta(\tilde{\mathbf{p}})}\right)+h_{2} v_{2} e^{\theta(\mathbf{p})}-h_{3} v_{3} e^{\theta(\tilde{\mathbf{p}})}\right|^{2}|x|^{2},
$$

where the diversity loss probability $P\left(\mu_{m}=0\right)$ is improved from $p_{e}^{2}$ to $p_{e}^{3}$.

Last but not least, phase rotations not only increase diversity and reduce the probability of diversity loss, but also reduce the error floor. The following Lemma derives the error floor of the generalized PPC scheme using phase rotations:

Lemma 3: The error floor of generalized PPC networking with phase rotations is given by

$$
P_{\mathrm{b}, N_{0, m} \rightarrow 0} \geq \frac{\frac{M K}{\widehat{M} \hat{K}} \log _{2}|X| \cdot p_{e}^{\hat{M} \hat{K}}+\log _{2}\lfloor S\rfloor \cdot p_{e}^{K M+d_{\min }}}{2\left(\frac{M K}{\widehat{M} \hat{K}} \log _{2}|X|+\log _{2}\lfloor S\rfloor\right)},
$$

The details of the derivations are provided in Appendix C. Compared with (35), the error floor of the QAM symbols remains the same, while the error floor due to the permutation vector is greatly reduced from $p_{e}^{2 d_{\min }}$ to $p_{e}^{K M+d_{\min }}$. This is due to the fact that, since the error floor occurs when diversity equals zero, i.e., $\sum_{m=1}^{M} \mu_{m}=0$, rotations yield nonzero $\mu_{m}$ when $p_{m}=\tilde{p}_{m}$ at $M-d_{\min }$ time instants. At these time instants, the probability of diversity loss is $P\left(\mu_{m}=0\right)=p_{e}^{K}$. Meanwhile, for those $d_{\min }$ time instants, comparing $\mu_{m}$ in (40) and (42), the probability of diversity loss is reduced after introducing rotations, which results into error floor reduction. As shown in Appendix $\mathrm{C}$, the lower bound of this diversity loss is reduced from $p_{e}^{2}$ to $p_{e}^{K+1}$, since at least $K+1$ paths are used to propagate $\mathbf{x}_{m}$ and $\tilde{\mathbf{x}}_{m}$ for $p_{m} \neq \tilde{p}_{m}$. The derivation shows that, by introducing phase rotations, the generalized PPC yields stronger robustness to erasures, thus also leading to throughput enhancement with a permutation set with small $d_{\min }$ and large $S$.

\section{Numerical Examples}

In this section, our theoretical analyses are validated to demonstrate the superior performance (better error rate performance, higher throughput, and stronger robustness to transmission outages) of generalized PPC combined with phase rotations in end-to-end networking of opportunistic links. We adopt a general end-to-end networking with $1 \leq L \leq 4$ and erasure model (2), with nodes capable of sensing link availability. For low-latency vehicular networks using proactive open-loop communication, the link number $L$ is generally 2. One can easily change to a different erasure model like (3) according to different node capabilities and network applications. These modifications only affect the value $p_{e}$, but the relative performances of different PPCs remain the same. Additionally, we assume a noise power spectral density independent of time, a waiting period equal to twice the number of links $W=2 L$, and $\hat{M}=M$ since the repetition along the time coordinate affects the QAM diversity. In [9], [11], the number of relay paths used to model the connections between the mobile users and the access points generally ranges between 3 and 5. Thus, in most of our numerical experiments we have chosen $R=4$. Nevertheless, as the concept of generalized PPC may be exploited for larger values of $R$, since higher power nodes in cellular network or other mobile users may be involved in the network in the future, we also simulate one case with $R=6$. Each link gain is assumed to be independent and identically distributed (i.i.d.) Rayleigh fading with normalized unit variance, and the erasure of different relay path is independent as well. For the decoder at the destination node, an exhaustive search is performed to jointly decode permutation vectors and QAM symbols, under the assumption that the erasures are perfectly identified. In [1], the maximum a posteriori (MAP) probability decoder that jointly decodes erasures, permutation vectors, and QAM symbols for conventional PPC transmission is investigated, and implemented by a low-complexity sphere decoder. Numerical simulations show that the error rate of this practical MAP decoder is close to what can be obtained by the exhaustive search used in this work. Finally, while the superiority of the conventional PPC over other techniques is demonstrated in [1], here we mainly focus on how generalized PPC outperforms the conventional one.

For the decoder at the destination node, an exhaustive search is performed to jointly decode the permutation vectors and the QAM symbols under the assumption that the erasures are 
perfectly known. In [1], the maximum a posteriori (MAP) probability decoder that jointly decodes erasures, permutation vectors, and QAM symbols for conventional PPC transmission is investigated, and implemented by a low-complexity sphere decoder. Numerical simulations show that the error rate of this practical MAP decoder is close to what can be obtained by the exhaustive search used in this work.

Numerical and analytical error rates are compared in Fig. 3. The scenarios with and without transmission outage are illustrated in Figs. 3(a) and 3(b), respectively. In Fig. 3(a), we set $d_{\min }=4$ and $2, S=4$ and $S=16$ permutation vectors for the transmission, respectively. The permutation set is generated using the two-phase greedy algorithm. For both point-to-point communication $(L=1)$ and multihop relay communication $(L=3)$, the numerical results (solid lines) tightly match analysis (dashed lines), thus validating the accuracy of our analyses. The performance is degraded as the number of links increases or the minimum Hamming distance decreases. Without erasures, the error rate can be vanishingly small at large SNR, while with transmission outages the error floor appears at $\eta=0.3$ as shown in Fig. 3(b). With $N=3$, the error floor occurs at higher SNR since the error rate curve has a lower slope than with $N=1$. Even with erasures, the accuracy of our analyses is confirmed by the agreement between theoretical and numerical results. As mentioned previously, the maximal diversity is dominated by $\min \left(\widehat{M}, d_{\text {min }}\right)$. In practice, the presence of erasures further reduces diversity, since certain paths are completely erased, and thus the error-curve slopes shown by the numerical results appear to be lower than with traditional communication systems with similar settings.

Fig. 4 compares three PPC schemes under the same throughput constraints and $M=4$. Specifically, the curves with circle markers refer to the conventional end-to-end PPC networking that accesses single relay paths $(K=1)$ and transmits single 16-QAM symbol; the curves with diamond markers refer to the generalized PPC scheme that simultaneously accesses two relay paths with path repetition of the QAM symbols, i.e., $(\widehat{K}, K)=(2,2)$ as in (13). Comparing these two schemes, since the generalized end-to-end PPC networking is allowed to access one more path, the number of usable permutation vectors increases from 24 to 360 , so that with the same size $S$ of the adopted permutation set the minimum Hamming distance increases from 2 to 3 , thus resulting in better error rate performance.

We also simulate the performance of the most general PPC scheme, which transmits different QPSK symbols through two paths $(\hat{K}, K)=(1,2)$ as in $(15)$, as shown by the curves with square markers. Comparing two different levels of generalizations of PPC, the one with $\hat{K}=1$ achieves 3 $\mathrm{dB}$ SNR gains. The reason is that, while the diversity of both schemes is equal to $\widehat{M}=M=4$, the scheme with $\widehat{K}=1$ splits the 16-QAM symbol into two QPSK symbols, which can be recovered more easily. This result validates our design guidelines for low erasure probability: While time repetition of QAM symbols is necessary for diversity gain, different paths should transmit QAM symbols as different as possible to improve the error rate by lowering the modulation alphabet, or to increase the throughput. Overall, the most general PPC scheme can improve performance by about $6 \mathrm{~dB}$ with respect to the conventional PPC scheme in our context. Note that actually the throughput of the generalized PPC can be larger than 2 bits per time instant, since more than 16 permutation vectors are generated by our algorithm. Nevertheless, for comparison purposes, we limit the size to $S=16$ for the generalized PPC in this case.

Fig. 5 demonstrates the performance of the generalized PPC for various throughputs and the improvement achieved by phase rotations with $R=4$ relay paths. For the former, taking the throughput 3.5 bits per time instant and $M=4$ as an example, this scheme transmits two 16-QAM symbols and adopts the permutation set with $S=64$ so that the other 6 bits are loaded on the permutation vectors with Hamming distance $d_{\min }=2$. For the conventional end-to-end PPC networking, at most $S \leq 24$ permutation vectors can be adopted to convey 4 bits. In this case, 1024-QAM modulation is required to transmit the remaining 10 bits, leading to poor error rate performance. For the systems that meets the throughput 4 bits per time instant, we adopt $M=2$ with a number of permutation vectors $S=16$, and transmit two QPSK constellation. The throughput can be further improve by applying higher QAM modulation in this case, while the diversity remains the same and thus the performance is still satisfactory at higher SNR. The results show that generalized PPCs can achieve higher throughput with satisfactory error rate performance in the end-to-end networking of opportunistic links. Note that while the conventional PPC only achieves better performance than the path-time code (PTC) [12], [20] under certain constraints, here we simulate the PTC (cyan curve) that simultaneously accesses two paths, uses two time instants, and provides 4 bits per time instant. Comparing with the generalized PPC having the same throughput (blue curves) and $(K, M)$, the latter achieves significantly better error rate performance. Although the former has larger $R=4$, other PPC users could access the same relay route by adopting permutation sets different from those of current user, i.e., adopting multiuser PPC [1]. Finally, since PTC applies coding to multiple QAM symbols, we believe that communication performance can be further improved by integrating PTC and PPC.

To examine the improvement achievable by phase rotations, we can see that, for target BER $10^{-3}$, SNR gains around 3 $\mathrm{dB}$ are achieved for systems with a 2.5 throughput constraint. Such improvement increases as SNR increases, indicating the diversity gain allowed by rotation. Additionally, note that the rotation effect is more pronounced for systems with low throughput constraints, since when fewer permutation vectors are adopted, their angle differences increase as reflected by (37). Thus, one can expect that for the 2 bits throughput constraint as in previous figure, the improvement caused by rotations becomes larger. Such results show that generalized PPC can provide higher throughput than the conventional PPC. By introducing rotations, the error rate can be further reduced.

Finally, Fig. 6 shows the error floor of generalized PPC with and without rotations. We increase the probability of link unavailability to 0.55 so that the error floor becomes significant. Comparing the curves with circle markers $(\hat{K}=1)$ to the 


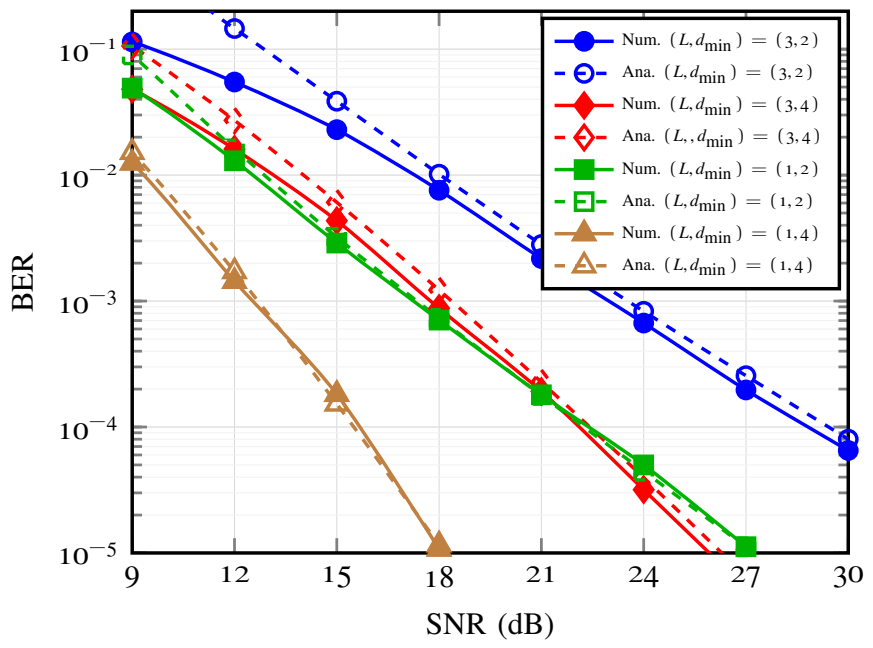

(a) Without opportunistic links.

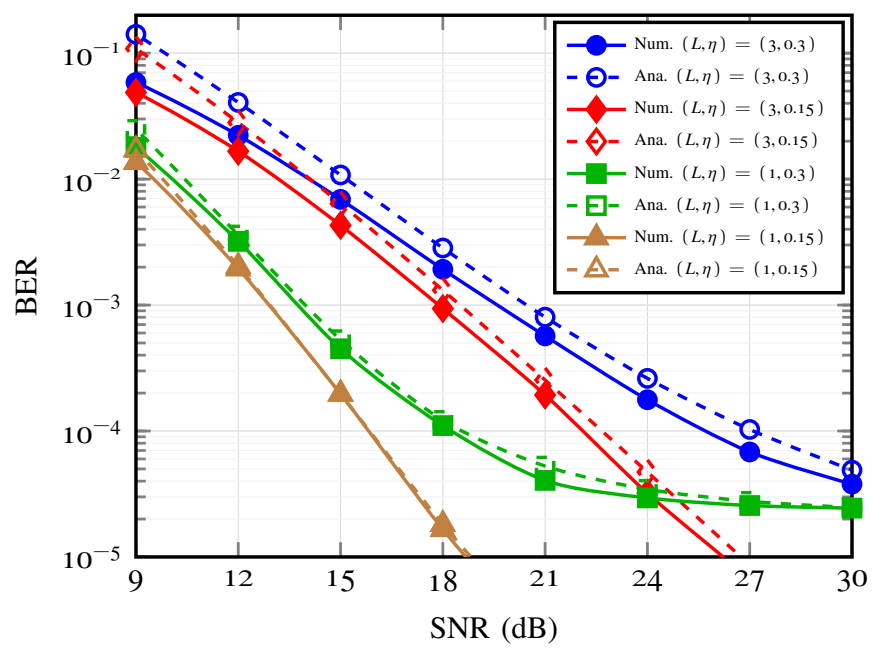

(b) With opportunistic links and $d_{\min }=4$.

Fig. 3. Comparison of numerical and analytical BERs of end-to-end PPC networking with QPSK and transmission parameters $(M, R, K, \widehat{K})=(4,6,2,2)$.

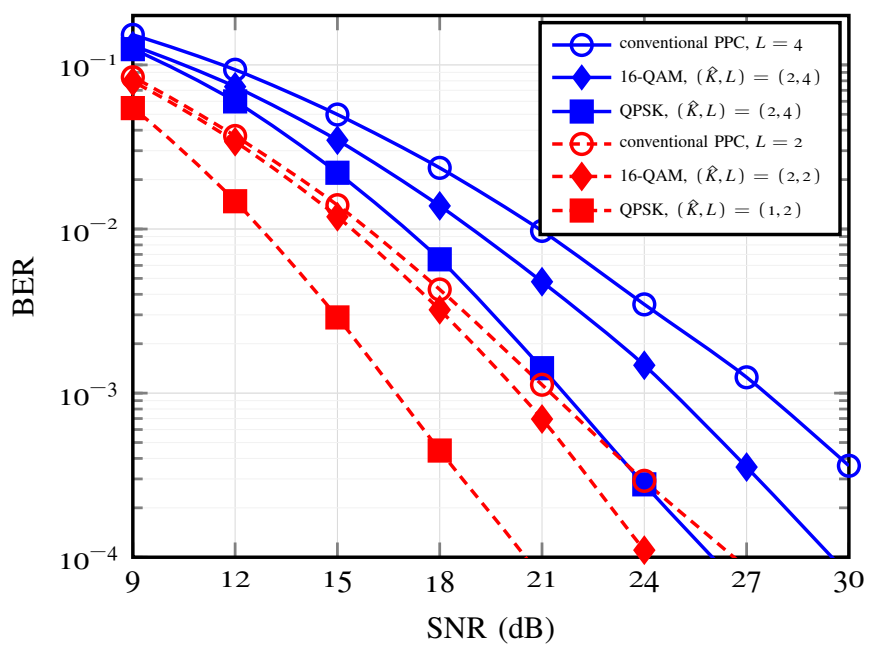

Fig. 4. BER comparisons of the conventional PPC (16-QAM, $d_{\min }=2, K=$ 1) and two generalized PPCs $\left(d_{\min }=3, K=2\right)$ under throughput constraint of 2 bits per time instant and $(M, R)=(4,4)$; the value $\eta=0.15$ is assumed.

other curves $(\hat{K}=2)$, it is seen that the repetition of QAM transmission along the path coordinate effectively reduces the error floor. This occurs because the error floor of the system with $\hat{K}=1$ is dominated by the QAM error floor $p_{e}^{\hat{K} \hat{M}}=p_{e}^{4}$. For the case with $\hat{R}=2$ and without rotations, the error floor is dominated by the permutation vectors, so that $p_{e}^{2 d_{\min }}=p_{e}^{6}$ or $p_{e}^{8}$ for different minimum Hamming distances, respectively. The curves with square markers refer to a system with rotations, which yield the lowest error floors. Specifically, the error floor of the PPC with $\hat{K}=1$ is around $10^{-2}$, while that of the PPC with $\hat{K}=2$ and rotations is around $5 \times 10^{-4}$, almost twenty times lower. For the case of $\left(d_{\min }, \hat{K}\right)=(3,2)$, rotations greatly improve the error floor, since, as we can see from (35) and (43), the exponent of the permutation error floor greatly increases from 6 to 11 . In this case, the error floor of the rotated PPC is again dominated by the QAM error floor $p_{e}^{8}$. Note that we have also depicted analytical error floors, computed by (35) and (43), to validate the accuracy of our analyses. The analytical results are slightly lower than the

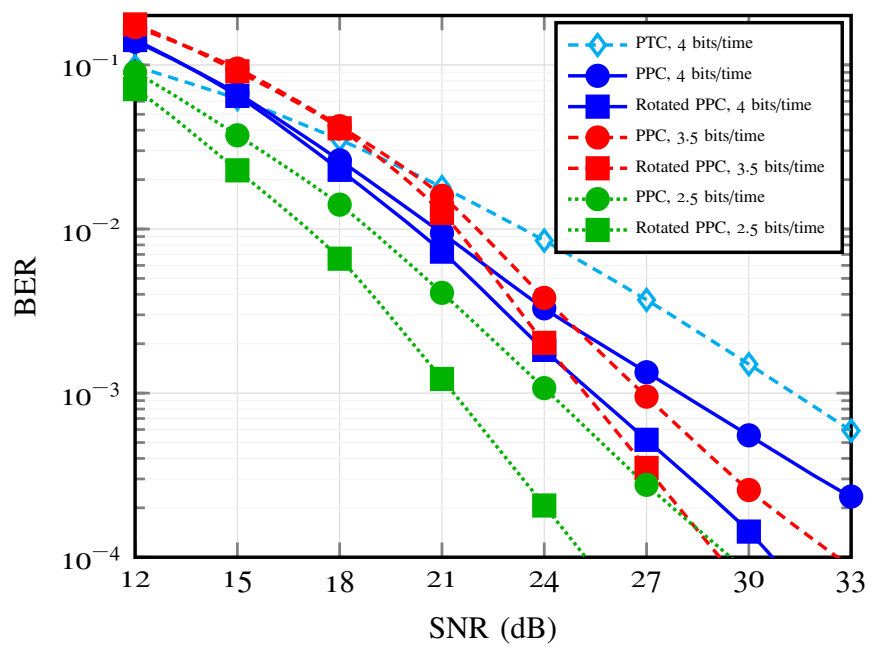

Fig. 5. BER comparisons of the generalized PPC schemes with and without rotation techniques for the various throughput scenarios; the transmission parameters $\left(R, K, \hat{K}, d_{\min }\right)=(4,2,1,2)$ and the scenarios $(L, \eta)=(4,0.15)$ are used. For the throughputs 4 bits per time instant, $M=2$ and QPSK are adopted. For the throughput 3.5 bits per time unit, $M=4$ and 16-QAM are adopted. For the throughputs 2.5 bits per time instant, $M=4$ and QPSK are adopted. The PTC achieving 4 bits per time instant using 16 QAM modulation and $(R, K, M)=(2,2,2)$ is also simulated.

numerical curves, because the lower bounds in Appendix B are used. The results illustrate the improvement made possible by rotations, which increase the robustness of generalized PPC to transmission outages. Moreover, our design guidelines for high erasure probability are validated, thus confirming that path repetition of QAM symbols is necessary to lower the error floor.

\section{Conclusions and Future Works}

In this work, we have generalized the introduction of PPC techniques for end-to-end networking of opportunistic links, a system with many applications like low-latency vehicular networks, energy-harvesting networks, and cognitive radio networks. The PPC investigated in this work is generalized by 


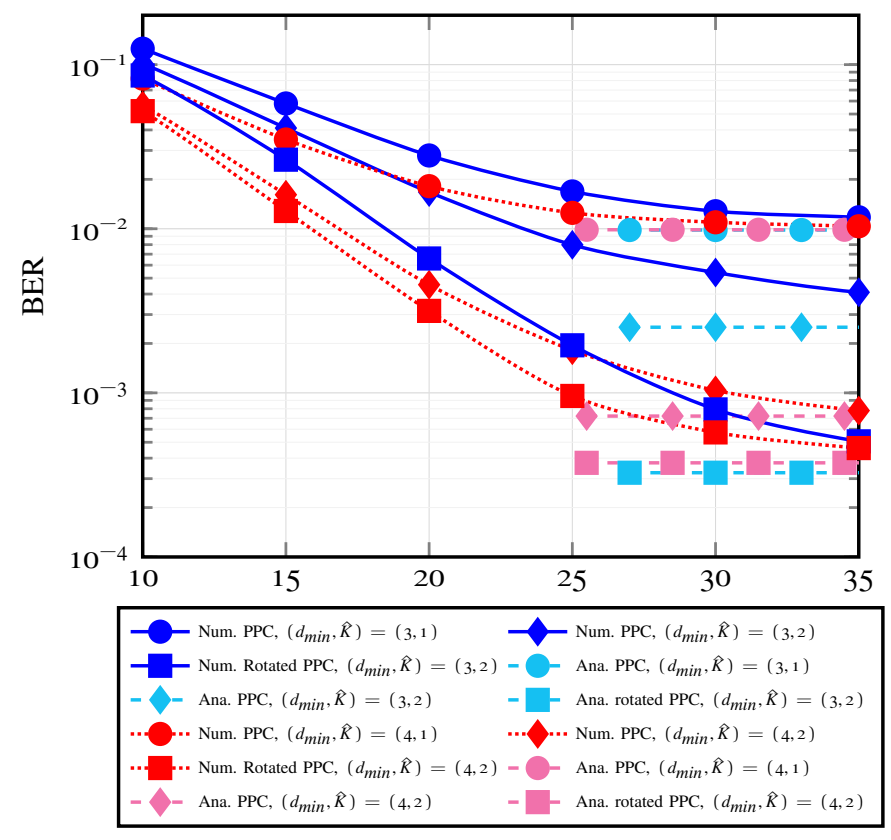

Fig. 6. Error Floor of the generalized end-to-end PPC networking which transmits same or different QAM symbols at the same time instant; QPSK, $(M, R, K)=(4,4,2)$ are used; The value chosen are $(L, \eta)=(3,0.55)$. The analytical error floors are also depicted.

introducing simultaneous access of multiple paths and transmission of multiple QAM symbols, which enhances error rate performance, throughput, and robustness to the erasures, and hence enables reliable end-to-end networking with open-loop communications. We propose an efficient algorithm to design the permutation sets for such generalized PPCs. Extensive theoretical analyses of error rate, diversity, and error floor shed light on the behavior of systems using generalized PPCs. In particular, QAM diversity is determined by time repetition, while permutation diversity equals the minimum Hamming distance of the adopted permutation set. Thus, to enhance the throughput or reduce the error rate, different QAM symbols should be transmitted through different relay paths, rather than at different time instants. Further, if the channel exhibits a high erasure probability, QAM repetition in the path coordinate is needed to reduce the error floor. Finally, adding phase rotations to generalized PPCs, the probability of a diversity loss and the error floor itself are decreased. Numerical results validate the accuracy of our analyses and show that generalized PPC can provide up to $6 \mathrm{~dB}$ SNR gains, which may be translated into higher throughputs. When phase rotations are added, further SNR gains and lower error floors can be achieved, implying that the generalized PPC with phase rotation is most robust to random transmission outages.

Several promising extensions of this work are possible. For example, the error floor and the diversity of the QAM symbols can be improved by applying sophisticated coding techniques for various QAM symbols, e.g., the combination of PPCs and PTC [12], [20]. Since the number of accessed paths is less than the number of multipath in the route, a generalized PPC can be extended as a multiple access technique as the conventional PPC [1]. Also, consideration of MAP decoder accounting for the erasures, the permutation vectors, and the QAM symbols may prove another interesting research direction.
APPENDIX

\section{A. Derivation of the $M G F$}

For notational simplicity, in the following derivation we omit the time index $m$. From (22), the random variable $\mu$ is given by

$$
\mu=\left|\sum_{k=1}^{K} h_{q_{k}} v_{q_{k}} x+\sum_{k^{\prime}=1}^{K} h_{\tilde{q}_{k^{\prime}}} v_{\tilde{q}_{k^{\prime}}} \tilde{x}\right|^{2} .
$$

By normalizing the variance of link gains, the path fading gain $h_{q_{k}}$ is an i.i.d. random variable formed by the product of $L$ unit-variance Rayleigh random variables. By defining $\beta_{L}$ as the square of the path fading gain, i.e., $\left|h_{q_{k}}\right|^{2}$, its probability density function (PDF) is expressed by the Meijer Gfunction (27) [37]. Therefore, the MGF of $\beta_{L}$ is given by [1]

$$
M_{\beta_{L}}(s)=G_{L, 1}^{1, L}\left[-s \mid \begin{array}{ccc}
0 & \ldots & 0 \\
& 0 &
\end{array}\right] .
$$

We now discuss four cases with at least one available path for either $x$ or $\tilde{x}$, and show that $\mu$ can be categorized by four kinds of random variables depending on the realizations of the erasures and the relationship between $q_{k}$ and $\tilde{q}_{k^{\prime}}$. Each case is represented or approximated by different forms of $\beta_{L}$, which allow their MGF to be computed using the Meijer G-function in (45).

1) Single available path for $x$ only: Since only $x$ is propagated through a single relay path, we have $\mu=\left|h_{q_{k}}\right|^{2}|x|^{2}, \exists k !$ : $v_{q_{k}}=1$, which is the square of a product of $L$ Rayleigh random variables, whose common PDF is the PDF of $|x|^{2} \beta_{L}$. The first kind of the MGF $M_{\mu}\left(\frac{s+s^{2}}{N_{0}}\right)$ is thus given by

$$
M_{\mu^{(1)}}\left(\frac{s+s^{2}}{N_{0}} \mid \mathbf{v}_{\mathbf{q}}, \mathbf{v}_{\tilde{\mathbf{q}}}\right)=G_{L, 1}^{1, L}\left[-\frac{\left(s+s^{2}\right)|x|^{2}}{N_{\mathrm{O}}} \mid \begin{array}{ccc}
0 & \ldots & 0 \\
& 0
\end{array}\right] .
$$

To average out the erasures, we need first to compute the conditional MGF. According to the explanation in Section IV, we have a total of $2 K-Z$ paths utilized by $x$ and $\tilde{x}$. Among them, $Z$ paths are jointly accessed by $x$ and $\tilde{x}$, while $K-Z$ paths accessed by either $x$ or $\tilde{x}$, respectively. Thus, the probability of single availability is computed as $p_{e}^{2 K-Z-1}\left(1-p_{e}\right)$, since only one of them is available with probability $p_{e}$, and the remaining paths are unavailable with probability $p e^{2 K-Z-1}$. Note that for various realizations of erasures in this case, their MGFs are identical, and thus we can compute the total probability of all the $K-Z$ events in this case:

$$
P_{\mu, 1}=(K-Z) \cdot p_{e}^{2 K-Z-1}\left(1-p_{e}\right),
$$

where the value $K-Z$ comes from choosing one path from $K-$ $Z$ relay paths used to propagate $x$.

2) Single available path for $\tilde{x}$ only: Similar to the previous case, since $\tilde{x}$ is propagated through a single relay path, we have $\mu=\left|h_{\tilde{q}_{k^{\prime}}}\right|^{2}|\tilde{x}|^{2}, \exists k^{\prime} !: v_{\tilde{q}_{k^{\prime}}}=1$, which is the square of a product of $L$ Rayleigh random variables as well. Therefore, the second kind of MGF $M_{\mu}\left(\frac{s+s^{2}}{N_{0}}\right)$ is given by

$M_{\mu^{(2)}}\left(\frac{s+s^{2}}{N_{0}} \mid \mathbf{v}_{\mathbf{q}}, \mathbf{v}_{\tilde{\mathbf{q}}}\right)=G_{L, 1}^{1, L}\left[-\frac{\left(s+s^{2}\right)|\tilde{x}|^{2}}{N_{0}} \mid \begin{array}{ccc}0 & \ldots & 0 \\ & 0\end{array}\right] \cdot|\tilde{x}|^{2}$. 
with the corresponding probability as (47)

$$
P_{\mu, 2}=(K-Z) \cdot p_{e}^{2 K-Z-1}\left(1-p_{e}\right),
$$

which is identical to (47).

3) Single available and identical path for $x$ and $\tilde{x}$ : In this case, both $x$ and $\tilde{x}$ are propagated by the same single path, i.e., $q_{k}=\tilde{q}_{k^{\prime}}$. The value of $\mu$ is simplified as $\left|h_{q_{k}}\right|^{2}|x-\tilde{x}|^{2}, \exists k !$ : $v_{q_{k}}=1, \exists k^{\prime} !: v_{\tilde{q}_{k^{\prime}}}=1, \quad q_{k}=\tilde{q}_{k^{\prime}}$, which is identically distributed as the random variable of $|x-\tilde{x}|^{2} \beta_{L}$. Therefore, the third kind of the MGF is thus derived as follows:

$M_{\mu^{(3)}}\left(\frac{s+s^{2}}{N_{0}} \mid \mathbf{v}_{\mathbf{q}}, \mathbf{v}_{\tilde{\mathbf{q}}}\right)=G_{L, 1}^{1, L}\left[-\frac{\left(s+s^{2}\right)|x-\tilde{x}|^{2}}{N_{0}} \mid \begin{array}{ccc}0 & \ldots & 0 \\ & 0 & \end{array}\right]$.

The corresponding probability is still $p_{e}^{2 K-Z-1}\left(1-p_{e}\right)$ as previous two cases. Nevertheless, since this available path is jointly accessed by $x$ and $\tilde{x}$, the accumulated probability of all the realizations of $\mathbf{v}_{q_{k}}$ and $\mathbf{v}_{\tilde{q}_{k^{\prime}}}$ in this case is given by:

$$
P_{\mu, 3}=Z \cdot p_{e}^{2 K-Z-1}\left(1-p_{e}\right),
$$

where $Z$ comes from choosing one path from $Z$ relay paths jointly accessed by both $x$ and $\tilde{x}$.

4) Multiple available paths for $x$ and $\tilde{x}$ : The last case is the most general one: here at least two different relay paths are available for the propagations of $x$ and $\tilde{x}$. Thus, (22) is reformulated by using three summations, corresponding to the available paths jointly accessed by $x$ and $\tilde{x}$, the events corresponding to the available path accessed by $x$ only, and the events corresponding to the available path accessed by $\tilde{x}$ only, respectively:

$$
\mu=\left|\sum_{\substack{\forall k \\ v_{q_{k}}=1, q_{k}=\tilde{q}_{k^{\prime}}}} h_{q_{k}}(x-\tilde{x})+\sum_{\substack{\forall k \\ v_{q_{k}}=1, q_{k} \neq \tilde{q}_{k^{\prime}}}} h_{q_{k}} x-\sum_{\substack{\forall k^{\prime} \\ v_{\tilde{q_{k}}}=1, \tilde{q}_{k^{\prime}} \neq q_{k}}} h_{\tilde{q}_{k^{\prime}}} \tilde{x}\right|^{2}
$$

At the RHS, the number of terms in the three summations are $Z^{(v)},\left\|\mathbf{v}_{\mathbf{q}}\right\|_{1}-Z^{(v)}$, and $\left\|\mathbf{v}_{\tilde{\mathbf{q}}}\right\|_{1}-Z^{(v)}$, respectively, where $Z^{(v)}$ denotes the number of total available paths and jointly accessed by $x$ and $\tilde{x}$ as defined in Sec. IV-A. In this case, $\mu$ is the square of the summation of products of $L$ Rayleigh random variables, whose PDF can be approximated by using the PDF of $\beta_{L-1}$ [1]. Thus, the fourth kind of the MGF takes the form of

$$
\begin{aligned}
& M_{\mu^{(4)}}\left(\frac{s+s^{2}}{N_{0}} \mid \mathbf{v}_{\mathbf{q}}, \mathbf{v}_{\tilde{\mathbf{q}}}\right) \approx G_{L-1,1}^{1, L-1} \\
& {\left[-\frac{s+s^{2}}{N_{\mathrm{o}}}\left(Z|x-\tilde{x}|^{2}+\left(\left\|\mathbf{v}_{\mathbf{q}}\right\|_{1}-Z\right)|x|^{2}+\left(\left\|\mathbf{v}_{\tilde{\mathbf{q}}}\right\|_{1}-Z\right)|\tilde{x}|^{2}\right) \mid \begin{array}{ll}
0 . . & 0 \\
& 0
\end{array}\right] .}
\end{aligned}
$$

Since the MGF is a function of $\left\|\mathbf{v}_{\mathbf{q}}\right\|_{1}$ and $\left\|\mathbf{v}_{\tilde{\mathbf{q}}}\right\|_{1}$, the associated probability is calculated individually by counting the number of available paths:

$$
P_{\mu_{m}^{(4)}}=p_{e}^{2 K-Z-\left\|\mathbf{v}_{\mathbf{q}}+\mathbf{v}_{\tilde{q}}\right\|_{1}}\left(1-p_{e}\right)^{\left\|\mathbf{v}_{\mathbf{q}}+\mathbf{v}_{\tilde{\mathbf{q}}}\right\|_{1}} .
$$

An individual summation for the fourth case is used in (26) to average the erasure realizations in this case. With $M_{\mu}\left(\frac{s+s^{2}}{N_{0}}\right)$ and the associated probability of all the four cases, we can compute the unconditional MGF $M_{\Lambda}(s)$ for the PEP derivation in (26), (28), and (29).

\section{B. Derivation of the Error Floor}

The error floor reflects the fact that at high SNR the destination node may fail to recover the data. Its probability is equivalent to the probability that $\bar{\Lambda}$ in (21) equals zero, i.e., $P_{b, N_{0, m} \rightarrow 0}=P(\bar{\Lambda}=0)=P\left(\sum_{m=1}^{M} \mu_{m}=0\right)$, implying that the transmission outage occurs at every time instant. We first derive the error floor of data conveyed by the QAM symbols, next of those conveyed by the permutation vectors. - The QAM symbol $x_{m, k}$ cannot be recovered even at infinitely large SNR if the erasures make $x_{m, k}$ and $\tilde{x}_{m, k}$ indistinguishable. The probability of this event is dominated by the probability that $\mathbf{p}=\tilde{\mathbf{p}}$, where the the random variable $\mu_{m}$ in (22) is rewritten as

$$
\mu_{m}=\left|\left(x_{m, k}-\tilde{x}_{m, k}\right) \sum_{k=1}^{\widehat{K}} h_{q_{m, k}} v_{q_{m, k}}\right|^{2}, \quad m=1, \ldots, \widehat{M}, \mathbf{p}=\tilde{\mathbf{p}},
$$

and thus we have $P\left(\mu_{m}=0\right)=p_{e}^{\widehat{R}}$. When the summation of all $\mu_{m}$ in (55) equal zero, meaning that the paths associated with $x_{m, k}$ and $\hat{x}_{m, k}$ for these $\hat{M}$ time instants are all erased, one cannot detect $x_{m, k}$, and hence the error floor of the QAM symbols shows up:

$$
P\left(\sum_{m=1}^{\hat{M}} \mu_{m}=0\right)=p_{e}^{\hat{R} \hat{M}}, \quad x_{m, k} \neq \tilde{x}_{m, k}, \mathbf{p}=\tilde{\mathbf{p}},
$$

Likewise, the error floor of the data conveyed by the permutation vectors is dominated by the event with $\mathbf{X}=\tilde{\mathbf{X}}$. In this case, we only have $d_{\min }$ nonzero $\mu_{m}$ at the time instants with $p_{m} \neq \tilde{p}_{m}$. Based on (52), these nonzero $\mu_{m}$ can be expressed in the form

$$
\mu_{m}=\left|\sum_{\substack{\forall k \\ q_{k} \neq \tilde{q}_{k^{\prime}}}} h_{q_{k}} v_{q_{k}} x_{m, k}-\sum_{\substack{\forall k^{\prime} \\ \tilde{q}_{k^{\prime}} \neq q_{k}}} h_{\tilde{q}_{k^{\prime}}}{\tilde{\tilde{q}^{\prime}}}_{\tilde{k}^{\prime}} x_{m, k^{\prime}}\right|^{2}, p_{m} \neq \tilde{p}_{m}, \mathbf{X}=\tilde{\mathbf{X}}
$$

where each summation includes those paths that are accessed by either $x_{m, k}$ or $\tilde{x}_{m, k}$, and thus comprises $K-Z_{m}$ erasures. The probability that $\mu_{m}=0$ is thus computed as

$$
P\left(\mu_{m}=0\right)=p_{e}^{2\left(K-Z_{m}\right)} \geq p_{e}^{2}, \quad p_{m} \neq \tilde{p}_{m},
$$

where the inequality comes from the fact that $\left(K-Z_{m}\right) \geq 1$. The error floor of the data conveyed by the permutation vector is thus given by

$$
P\left(\sum_{\substack{\forall m \\ p_{m} \neq \tilde{p}_{m}}} \mu_{m}=0\right)=\prod_{\substack{\forall m \\ p_{m} \neq \tilde{p}_{m}}} p_{e}^{2\left(K-Z_{m}\right)} \geq p_{e}^{2 d_{\min }}, \mathbf{p} \neq \tilde{\mathbf{p}}, \mathbf{X}=\tilde{\mathbf{X}}
$$

The overall error floor is the linear combination of (56) and (59), weighted by the number of bits conveyed by the QAM symbols $\left(N \log _{2}|\chi|\right)$ and by the permutation vector $\left(\log _{2} S\right)$ respectively. Multiplying by 0.5 , the error probability of randomly guessing the binary bit value, we have the error floor of the generalized end-to-end PPC networking in (35). 


\section{Derivation of the Error Floor with Phase Rotations}

With phase rotations, the error floor of the QAM symbols remains the same as in (56). As for the permutation vector, the major difference is that, since $\mathbf{x}_{m}$ and $\tilde{\mathbf{x}}_{m}$ becomes $\mathbf{x}_{m} e^{\theta(\mathbf{p})}$ and $\tilde{\mathbf{x}}_{m} e^{\theta(\tilde{\mathbf{p}})}$, we have nonzero $\mu_{m}$ for all the time instants even with $\mathbf{X}=\tilde{\mathbf{X}}$. Specifically, at the time instants with $p_{m} \neq \tilde{p}_{m}, \mu_{m}$ is represented as:

$\left.\mu_{m}=\mid \sum_{\substack{\forall k \\ q_{k}=\tilde{q}_{k^{\prime}}}} h_{q_{k}} v_{q_{k}} x_{m, k}+\sum_{\substack{\forall k \\ q_{k} \neq \tilde{q}_{k^{\prime}}}} h_{q_{k}} v_{q_{k}} x_{m, k}+\sum_{\substack{\forall k^{\prime} \\ \tilde{q}_{k^{\prime}} \neq q_{k}}} h_{\tilde{q}_{k^{\prime}}}{\tilde{\tilde{q}^{\prime}}}_{k^{\prime}} x_{m, k^{\prime}}\right)\left.\right|^{2}$,

for $p_{m} \neq \tilde{p}_{m}, \mathbf{X}=\tilde{\mathbf{X}}$, where compared with (57), we have an additional first summation in the RHS that contains $Z_{m}$ terms. Thus we should rewrite as

$$
P\left(\mu_{m}=0\right)=p_{e}^{Z_{m}+2\left(K-Z_{m}\right)}=p_{e}^{2 K-Z_{m}} \geq p_{e}^{K+1}, \quad p_{m} \neq \tilde{p}_{m},
$$

where the inequality comes from the fact that $\left(2 K-Z_{m}\right)=$ $\left(K+\left(K-Z_{m}\right)\right) \geq(K+1)$. For the time instants with $p_{m}=\tilde{p}_{m}$, the value of $\mu_{m}$ is changed from zero to

$$
\mu_{m}=\left|\left(x_{m, k} e^{\theta(\mathbf{p})}-\tilde{x}_{m, k} e^{\theta(\tilde{\mathbf{p}})}\right) \sum_{k=1}^{\hat{R}} h_{q_{m, k}} v_{q_{m, k}}\right|^{2}, p_{m}=\tilde{p}_{m}, \mathbf{X}=\tilde{\mathbf{X}},
$$

This is equal to zero when all the $K$ paths are erased, which occurs with probability $p_{e}^{K}$. Therefore, compared with (59), the error floor of the permutation vectors for the generalized endto-end PPC networking with rotation technique is represented as

$$
P\left(\sum_{m=1}^{M} \mu_{m}=0\right) \geq\left(p_{e}^{K}\right)^{M-d_{\min }} \cdot\left(p_{e}^{K+1}\right)^{d_{\min }}=p_{e}^{K M+d_{\min }}
$$

for $\mathbf{p} \neq \tilde{\mathbf{p}}, \mathbf{X}=\tilde{\mathbf{X}}$, which depends on the size of the used path-time plane and the minimum Hamming distance. By combining (56) and (63), and following the same procedure, the error floor of the PPC with phase rotations (43) can be derived.

\section{REFERENCES}

[1] I.-W. Lai, C.-H. Lee, K.-C. Chen, and E. Biglieri, "Path-permutation codes for end-to-end transmission in ad hoc cognitive radio networks," IEEE Trans. Wireless Commun., vol. 14, no. 6, pp. 3309-3321, 2015.

[2] Z. Zhang, H. Wu, H. Zhang, H. Dai, and N. Kato, "Virtual-mimo-boosted information propagation on highways," IEEE Wireless Commun., vol. 15, no. 2, pp. 1420-1431, Feb 2016.

[3] K.-C. Chen, T. Zhang, R. D. Gitlin, and G. Fettweis, "Ultra-low latency mobile networking," IEEE Network, vol. 33, no. 2, pp. 181-187, Mar 2019.

[4] X. Wu, S. Subramanian, R. Guha, R. G. White, J. Li, K. W. Lu, A. Bucceri, and T. Zhang, "Vehicular communications using dsrc: Challenges, enhancements, and evolution," IEEE J. Sel. Areas Commun., vol. 31, no. 9, pp. 399-408, Sep. 2013.

[5] M. A. Lema, A. Laya, T. Mahmoodi, M. Cuevas, J. Sachs, J. Markendahl, and M. Dohler, "Business case and technology analysis for $5 \mathrm{~g}$ low latency applications," IEEE Access, vol. 5, pp. 5917-5935, Apr. 2017.

[6] S.-Y. Lien, S.-C. Hung, and K.-C. Chen, "Optimal radio access for fully packet-switching 5g networks," in Proc. IEEE Int. Conf. Commun. (ICC), June 2015, pp. 3921-3926.
[7] R. H. Y. Louie, M. R. McKay, and I. B. Collings, "Open-loop spatial multiplexing and diversity communications in ad hoc networks," IEEE Trans. Inf. Theory, vol. 57, no. 1, pp. 317-344, Jan 2011.

[8] S.-C. Hung, X. Zhang, A. Festag, K.-C. Chen, and G. Fettweis, "Vehicle-centric network association in heterogeneous vehicle-to-vehicle networks," IEEE Trans. Veh. Technol., vol. 68, no. 6, pp. 5981-5996, June 2019.

[9] C.-H. Zeng and K.-C. Chen, "Downlink multiuser detection in the virtual cell-based ultra-low latency vehicular networks," IEEE Trans. Veh. Technol., vol. 68, no. 5, pp. 4651-4666, May 2019.

[10] S.-C. Hung, H. Hsu, S.-M. Cheng, Q. Cui, and K.-C. Chen, "Delay guaranteed network association for mobile machines in heterogeneous cloud radio access network," IEEE Trans. Mobile Comput., vol. 17, no. 12, pp. 2744-2760, Dec. 2018.

[11] C.-Y. Lin, K.-C. Chen, D. Wickramasuriya, S.-Y. Lien, and R. D. Gitlin, "Anticipatory mobility management by big data analytics for ultra-low latency mobile networking," in Proc. IEEE Int. Conf. Commun. (ICC), May 2018, pp. 1-7.

[12] I.-W. Lai, C.-H. Lee, and K.-C. Chen, "A virtual MIMO path-time code for cognitive ad hoc networks," IEEE Commun. Lett., vol. 17, no. 1, pp. 4-7, Jan. 2013.

[13] P. Djukic and S. Valaee, "Reliable packet transmissions in multipath routed wireless networks," IEEE Trans. Mobile Comput., vol. 5, no. 5, pp. 548-559, May 2006.

[14] E. Başar, M. Wen, R. Mesleh, M. D. Renzo, Y. Xiao, and H. Haas, "Index modulation techniques for next-generation wireless networks," IEEE Access, vol. 5, pp. 16693-16746, Aug. 2017.

[15] R. Y. Mesleh, H. Haas, S. Sinaović, C. W. Ahn, and S. Yun, "Spatial modulation," IEEE Trans. Veh. Technol., vol. 57, no. 4, pp. 2228-2241, Jul. 2008.

[16] I.-W. Lai, J.-W. Shih, C.-W. Lee, H.-H. Tu, J.-C. Chi, J.-S. Wu, and Y.H. Huang, "Spatial permutation modulation for multiple-input multipleoutput (mimo) systems," IEEE Access, vol. 7, pp. 68 206-68 218, 2019.

[17] P.-Y. Chen, W.-C. Ao, and K.-C. Chen, "Rate-delay enhanced multipath transmission scheme via network coding in multihop networks," IEEE Commun. Lett., vol. 16, no. 3, pp. 281-283, Mar. 2012.

[18] M. K. Marina and S. R. Das, "On-demand multipath distance vector routing in ad hoc networks," in Proc. 2001 IEEE Int. Conf. on Network Protocol (ICNP), Nov. 2001, pp. 14-23.

[19] S. Fashandi, S. O. Gharan, and A. K. Khandani, "Path diversity over packet switched networks: Performance analysis and rate allocation," IEEE/ACM Trans. Netw., vol. 18, no. 5, pp. 1373-1386, May 2010.

[20] I.-W. Lai, C.-L. Chen, C.-H. Lee, K.-C. Chen, and E. Biglieri, "End-toend virtual MIMO Transmission in ad hoc cognitive radio networks," IEEE Trans. Wireless Commun., vol. 13, no. 1, pp. 330-341, Jan. 2014.

[21] I.-W. Lai, C.-H. Lee, K.-C. Chen, and E. Biglieri, "Open-loop end-to-end transmission for multihop opportunistic networks with energy-harvesting devices," IEEE Trans. Commun., vol. 64, no. 7, pp. 2860-2872, 2016.

[22] P. Djukic and S. Valaee, "Reliable packet transmission in multipath routed wireless networks," IEEE Trans. Mobile Comput., vol. 5, no. 5, pp. 548-559, May 2006.

[23] D. Saha, S. Roy, S. Bandyopadhyay, T. Ueda, and S. Tanaka, "An adaptive framework for multipath routing via maximally zone-disjoint shortest paths in ad hoc wireless networks with directional antenna," in Proc. IEEE Glob. Comm. Conf. (GLOBECOM), Dec. 2003, pp. 226-230.

[24] Y.-H. Wang, H.-Z. Lin, and S.-M. Chang, "Interfering-aware QoS multipath routing for ad hoc wireless network," in Proc. IEEE Int. Conf. Advanced Information Networking and Applications, 2004, pp. 29-34.

[25] S. Ju and J. B. Evans, "Cognitive multipath multi-channel routing protocol for mobile ad-hoc networks," in Proc. IEEE Glob. Comm. Conf. (GLOBECOM), Dec. 2003, pp. 1-6.

[26] R. G. Jaber and J. G. Andrews, "A lower bound on the capacity of wireless erasure networks," IEEE Trans. Inf. Theory, vol. 57, no. 10, pp. 6502-6513, Oct 2011.

[27] M. G. Khoshkholgh, K. Navaie, and H. Yanikomeroglu, "On the impact of the primary network activity on the achievable capacity of spectrum sharing over fading channels," IEEE Trans. Wireless Commun., vol. 8, no. 4, pp. 2100-2111, Apr. 2009.

[28] E. Biglieri, Coding for Wireless Channels. New York, NY: Springer, 2005.

[29] H. C. Ferreira, A. J. H. Vinck, T. G. Swart, and I. de Beer, "Permutation trellis codes," IEEE Trans. Commun., vol. 53, no. 11, pp. 1782-1789, Nov. 2005.

[30] Y.-J. Lai, "Hierarchical optimization: A satisfactory solution," Fuzzy Sets Syst., vol. 77, no. 3, pp. 321-335, Feb. 1996. 
[31] P.-Y. Chen, Y.-H. Chiang, Y.-A. Chu, Z.-S. Yang, and I.-W. Lai, "Theoretical bound of permutation set size for path-permutation code and spatial permutation modulation," in preparation.

[32] G. Caire, G. Taricco, and E. Biglieri, "Bit-interleaved coded modulation," IEEE Trans. Inf. Theory, vol. 44, no. 3, pp. 927-946, May 1998.

[33] A. Martinez, A. Guillén i Fàbregas, and G. Caire, "Error probability analysis of bit-interleaved coded modulation," IEEE Trans. Inf. Theory, vol. 52, no. 1, pp. 262-271, Jan. 2006.

[34] E. Biglieri, G. Caire, G. Taricco, and J. Ventura-Traveset, "Simple method for evaluating error probabilities," IEE Electron. Lett., vol. 32, no. 3, pp. 191-192, Feb. 1996.

[35] M. Simon and M. S. Alouini, Digital Communications over Fading Channels. John Wiley \& Sons, 2005.

[36] I.-W. Lai, C.-H. Lee, K.-C. Chen, and E. Biglieri, "Performance of pathtime codes for end-to-end transmission in ad hoc multihop networks," in Proc. IEEE Int. Symp. Inform. Theory (ISIT), June 2014, pp. 66-70.

[37] J. Salo, H. M. El-Sallabi, and P. Vainkainen, "The distribution of the product of independent Rayleigh random variables," IEEE Trans. Antennas Propag., vol. 54, no. 2, pp. 639-643, Feb. 2006. 